University of Texas Rio Grande Valley

ScholarWorks @ UTRGV

$1-10-2013$

\title{
Limits on the stochastic gravitational wave background from the North American Nanohertz Observatory for Gravitational Waves
}

\author{
P. B. Demorest \\ R. D. Ferdman \\ M. E. Gonzalez \\ D. Nice \\ S. Ransom
}

See next page for additional authors

Follow this and additional works at: https://scholarworks.utrgv.edu/pa_fac

Part of the Astrophysics and Astronomy Commons

\section{Recommended Citation}

P. B. Demorest, et. al., (2013) Limits on the stochastic gravitational wave background from the North American Nanohertz Observatory for Gravitational Waves.Astrophysical Journal762:2. DOI: http://doi.org/ $10.1088 / 0004-637 X / 762 / 2 / 94$

This Article is brought to you for free and open access by the College of Sciences at ScholarWorks @ UTRGV. It has been accepted for inclusion in Physics and Astronomy Faculty Publications and Presentations by an authorized administrator of ScholarWorks @ UTRGV. For more information, please contact justin.white@utrgv.edu, william.flores01@utrgv.edu. 


\section{Authors}

P. B. Demorest, R. D. Ferdman, M. E. Gonzalez, D. Nice, S. Ransom, I. H. Stairs, Z. Arzoumanian, A. Brazier, S. Burke-Spolaor, S. J. Chamberlin, J. M. Cordes, J. Ellis, L. S. Finn, P. Freire, S. Giampanis, F. Jenet, V. M. Kaspi, J. Lazio, A. N. Lommen, M. McLaughlin, N. Palliyaguru, D. Perrodin, R. M. Shannon, X. Siemens, D. Stinebring, J. Swiggum, and W. W. Zhu 


\title{
LIMITS ON THE STOCHASTIC GRAVITATIONAL WAVE BACKGROUND FROM THE NORTH AMERICAN NANOHERTZ OBSERVATORY FOR GRAVITATIONAL WAVES
}

\author{
P. B. Demorest ${ }^{1}$, R. D. Ferdman ${ }^{2}$, M. E. Gonzalez ${ }^{3}$, D. Nice ${ }^{4}$, S. Ransom ${ }^{1}$, I. H. Stairs ${ }^{3}$, Z. Arzoumanian ${ }^{5,6}$, A. Brazier ${ }^{7}$, \\ S. Burke-Spolaor ${ }^{8}$, S. J. Chamberlin ${ }^{9}$, J. M. Cordes $^{7}$, J. Ellis ${ }^{9}$, L. S. Finn ${ }^{10}$, P. Freire ${ }^{11}$, S. Giampanis ${ }^{9}$, F. Jenet ${ }^{12}$, \\ V. M. Kaspi ${ }^{2}$, J. Lazio ${ }^{8}$, A. N. Lommen ${ }^{13}$, M. McLaughlin ${ }^{14}$, N. Palliyaguru ${ }^{14}$, D. Perrodin ${ }^{13}$, R. M. ShannON ${ }^{15}$, \\ X. Siemens ${ }^{9}$, D. Stinebring ${ }^{16}$, J. Swiggum ${ }^{14}$, And W. W. Zhu ${ }^{3}$ \\ ${ }^{1}$ National Radio Astronomy Observatory, 520 Edgemont Road, Charlottesville, VA 22903, USA \\ 2 Department of Physics, McGill University, 3600 rue Universite, Montreal, QC H3A 2T8, Canada \\ ${ }^{3}$ Department of Physics and Astronomy, University of British Columbia, 6224 Agricultural Road, Vancouver, BC V6T 1Z1, Canada \\ ${ }^{4}$ Department of Physics, Lafayette College, Easton, PA 18042, USA \\ ${ }^{5}$ Center for Research and Exploration in Space Science and Technology and X-Ray Astrophysics Laboratory, NASA Goddard Space Flight Center, \\ Code 662, Greenbelt, MD 20771, USA \\ ${ }^{6}$ Universities Space Research Association, Columbia, MD 21044, USA \\ ${ }^{7}$ Department of Astronomy, Cornell University, Ithaca, NY 14853, USA \\ ${ }^{8}$ Jet Propulsion Laboratory, California Institute of Technology, 4800 Oak Grove Drive, Pasadena, CA 91106, USA \\ ${ }^{9}$ Center for Gravitation, Cosmology and Astrophysics, Department of Physics, University of Wisconsin-Milwaukee, P.O. Box 413, Milwaukee, WI 53201, USA \\ ${ }^{10}$ Department of Astronomy and Astrophysics, Pennsylvania State University, University Park, PA 16802, USA \\ ${ }^{11}$ Max-Planck-Institut fur Radioastronomie, D-53121 Bonn, Germany \\ ${ }^{12}$ Center for Gravitational Wave Astronomy, University of Texas at Brownsville, Brownsville, TX 78520, USA \\ ${ }^{13}$ Department of Physics and Astronomy, Franklin and Marshall College, P.O. Box 3003, Lancaster, PA 17604, USA \\ ${ }^{14}$ Department of Physics, West Virginia University, P.O. Box 6315, Morgantown, WV 26505, USA \\ ${ }^{15}$ CSIRO Astronomy and Space Science, Box 76, Epping NSW 1710, Australia \\ ${ }^{16}$ Department of Physics and Astronomy, Oberlin College, Oberlin, OH 44074, USA \\ Received 2012 March 7; accepted 2012 November 15; published 2012 December 19
}

\begin{abstract}
We present an analysis of high-precision pulsar timing data taken as part of the North American Nanohertz Observatory for Gravitational Waves (NANOGrav) project. We have observed 17 pulsars for a span of roughly five years using the Green Bank and Arecibo radio telescopes. We analyze these data using standard pulsar timing models, with the addition of time-variable dispersion measure and frequency-variable pulse shape terms. Sub-microsecond timing residuals are obtained in nearly all cases, and the best rms timing residuals in this set are $\sim 30-50 \mathrm{~ns}$. We present methods for analyzing post-fit timing residuals for the presence of a gravitational wave signal with a specified spectral shape. These optimally take into account the timing fluctuation power removed by the model fit, and can be applied to either data from a single pulsar, or to a set of pulsars to detect a correlated signal. We apply these methods to our data set to set an upper limit on the strength of the $\mathrm{nHz}$-frequency stochastic supermassive black hole gravitational wave background of $h_{c}\left(1 \mathrm{yr}^{-1}\right)<7 \times 10^{-15}(95 \%)$. This result is dominated by the timing of the two best pulsars in the set, PSRs J1713+0747 and J1909-3744.
\end{abstract}

Key words: gravitational waves - methods: data analysis - pulsars: general - pulsars: individual (J0030+0451, J0613-0200, J1012+5307, J1455-3330, J1600-3053, J1640+2224, J1643-1224, J1713+0747, J1744-1134,

J1853+1308, B1855+09, J1909-3744, J1910+1256, J1918-0642, B1953+29, J2145-0750, J2317+1439)

\section{INTRODUCTION}

The direct detection of gravitational radiation (or gravitational waves $(\mathrm{GW})$ ) is currently a major goal in experimental physics. As described by general relativity, GW are freely propagating wave solutions to Einstein's equation. Detecting GW would confirm another key prediction of general relativity. GW are expected to be generated by nearly any configuration of accelerating mass, although due to the weakness of gravity, large masses or high accelerations are required to radiate significant GW. This means that astronomical objects are the only sources expected to produce measurable $\mathrm{GW}$, and that we can in turn use these detections to learn about the GW sources themselves; GW astronomy will provide an entirely new window through which we can view the universe. Binary systems are expected to account for a large fraction of the detectable GW signals, but we cannot discount "exotic" or unexpected sources either.

One of the best tools we have for making precise astronomical measurements is the timing of pulses from radio pulsars. The pulse times of arrival can be analyzed via a model that counts every single rotation of the star over years or even decades.
This provides detailed information about the neutron star itself (via spin-down rate and spin irregularities), its binary orbit (via orbital Doppler and relativistic effects), and astrometry. Timing of double-neutron star binary systems has already provided strong evidence for the existence of GW, through measurements of orbital evolution induced by the generation of GW by the binary system (Taylor \& Weisberg 1989; Weisberg et al. 2010). The medium through which the radio pulses travel from the pulsar to Earth also affects the signal. This has been used extensively to probe the ionized component of the interstellar medium in a variety of ways (e.g., Rickett 1990). The presence of GW along the line of sight also will affect the pulse travel times. This forms the basis for the use of pulsars as gravitational wave detectors.

The influence of GW on pulsar timing, and its potential use in GW detection, was first noted over 30 years ago (Sazhin 1978; Detweiler 1979). A major step forward was provided by Hellings \& Downs (1983), who showed that a GW signal will produce correlated timing fluctuations in a set of pulsars, a concept that came to be known as a pulsar timing array (PTA; Foster \& Backer 1990). A second major advance was the 
discovery of millisecond pulsars (Backer et al. 1982) — the high spin rate and stability of these objects improves GW sensitivity by orders of magnitude compared with canonical $\sim 1$ s pulsars. Pulsar timing is most sensitive to $\mathrm{GW}$ with period comparable to the observational time span, typically $1-10$ years, so the GW frequency is in the nanohertz band. In this frequency range the brightest expected sources are supermassive black hole (SMBH) binaries, which may be visible either as a stochastic background (e.g., Jaffe \& Backer 2003; Sesana et al. 2008) or as individual sources (Sesana et al. 2009). A number of previous upper limits have been placed (Kaspi et al. 1994; Jenet et al. 2004, 2006; van Haasteren et al. 2011), but no successful detection has yet been accomplished. The topic continues to attract attention and a number of dedicated PTA research projects are now active worldwide.

In this paper, we present new high-precision timing observations of 17 millisecond pulsars, covering a five-year time span. These data were obtained as part of the North American Nanohertz Observatory for Gravitational Waves (NANOGrav) project, ${ }^{17}$ using the two largest single-dish telescopes available, Arecibo Observatory and the NRAO Green Bank Telescope (GBT). In Section 2 we describe the observational setup and data set. In Section 3 we present our methods and results for the determination of timing models. In Section 4 we present a timedomain algorithm for measuring the cross-correlation between pairs of post-fit residuals, and apply this to our timing data to place limits on the stochastic gravitational wave background. We discuss the astrophysical implications of these results in Section 5 and summarize our findings in Section 6.

\section{OBSERVATIONS}

The observations presented here were carried out over a fiveyear period, from 2005 to 2010, as part of a program specifically designed to measure or constrain the $\mathrm{nHz}$-frequency stochastic gravitational wave background. The sources were observed with one or both of the $305 \mathrm{~m}$ NAIC Arecibo Observatory or the $100 \mathrm{~m}$ NRAO GBT, with a typical observational cadence of four to six weeks between sessions (see Figure 1). Scheduling at each observatory was independent, and the sessions were typically not performed simultaneously at the two telescopes.

These sources were selected from the known population of bright millisecond pulsars (MSPs), excluding those found in globular clusters. Source selection was performed with the goal of obtaining the highest timing precision possible, to maximize sensitivity to gravitational waves. Due to its higher gain, Arecibo was used to observe all sources in its visible portion of the sky $\left(\sim 0^{\circ}-38^{\circ}\right.$ declination $)$, while sources outside this range were observed with the GBT, down to its minimum declination of $\sim-45^{\circ}$. One source presented in this paper, J1713+0747, was observed with both telescopes. ${ }^{18}$

Each pulsar was observed at two widely separated radio frequencies in order to track variation in dispersion measure (DM; see Section 3.2). As the different frequency measurements use different receivers, these observations are also not simultaneous. The choice of receivers used to observe any given pulsar was determined from the availability and performance of the receiver systems at each telescope, and from the spectral index

\footnotetext{
17 http://www.nanograv.org

18 Another bright MSP, PSR B1937+21, is also observed with both telescopes as part of this project. We have for now excluded it from this analysis due to its well-documented high levels of spin noise (e.g., Kaspi et al. 1994) and interstellar medium systematics (e.g., Ramchandran et al. 2006).
}

of the pulsar. At Arecibo, multi-frequency data are obtained in a single observing session, within $\sim 1 \mathrm{hr}$ for each source. At the GBT, the different frequencies were observed up to $\sim 1$ week apart. In a given epoch the observing time per frequency band for each source was anywhere from 15 to 45 minutes, with shorter integration times in general at Arecibo. The telescopes and frequencies used to observe each source, along with its basic physical parameters such as spin period and DM, are listed in Table 1. Three pulsars in this set (PSRs J1853+1308, $\mathrm{J} 1910+1256$, and B1953+29) were originally observed as part of a different project at Arecibo (Gonzalez et al. 2011) and consequently only have single-band data for most of the time span. All receiver systems used at $800 \mathrm{MHz}$ and above are sensitive to orthogonal linear polarizations. The $430 \mathrm{MHz}$ system at Arecibo is sensitive to dual circular polarizations.

At each observatory, site time is kept by a local hydrogenmaser-based clock. Clock comparison systems monitor and record the difference between site time and the GPS time signal. We use these records to later correct pulse times of arrival first from site time to GPS time, then to the "retrospective" realization of Terrestrial Time produced by the Bureau International des Poids et Mesures (e.g., Guinot 1988). The analysis presented here uses the 2011 version of the timescale, also known as TT(BIPM11).

All observations were performed using the identical Astronomical Signal Processor (ASP) and Green Bank Astronomical Signal Processor (GASP) pulsar backend systems (Demorest 2007) at Arecibo and the GBT, respectively. These systems perform real-time coherent dedispersion, full-Stokes detection, and pulse period folding in software, using 20-node Linuxbased computer clusters. Channelized voltage data are supplied to the cluster from a SERENDIP-V FPGA board ${ }^{19}$ controlled by a Compact PCI single-board computer. The SERENDIP-V board performs analog-to-digital conversion and a digital polyphase filterbank operation to split a $128 \mathrm{MHz}$ bandwidth, dual-polarization signal input from the telescope into $324 \mathrm{MHz}$ wide channels. Both the initial digitization of the signal and the channelized output use 8 bit complex quantization. Further details of the hardware and real-time software used in these observations were described by Demorest (2007). While the $4 \mathrm{MHz}$ channelization provides a minimum time resolution of $250 \mathrm{~ns}$, the final time resolution depends on the pulse period and number of profile bins used to average the profile-in this case, either 2048 (GBT) or 4096 (Arecibo) bins were used. Profiles were typically integrated for one minute (Arecibo) or three minutes (GBT). The total bandwidth processed varied with pulsar and observing frequency, limited by either the real-time computational load or the receiver bandpass. A maximum of $64 \mathrm{MHz}$ was used in most cases, with smaller bandwidths down to $\sim 20 \mathrm{MHz}$ used for low-frequency or high-DM observations.

\section{TIMING ANALYSIS}

In this section, we describe the procedures used in the timing portion of the data analysis. These can be split into two main areas: analysis dealing with pulse profiles, including polarization calibration and determination of pulse times of arrival (Section 3.1); and fitting a physical timing model to the arrival times (Section 3.2). The further analysis step of determining GW background limits is discussed later, in Section 4 .

\footnotetext{
19 https://casper.berkeley.edu/galfa/
} 


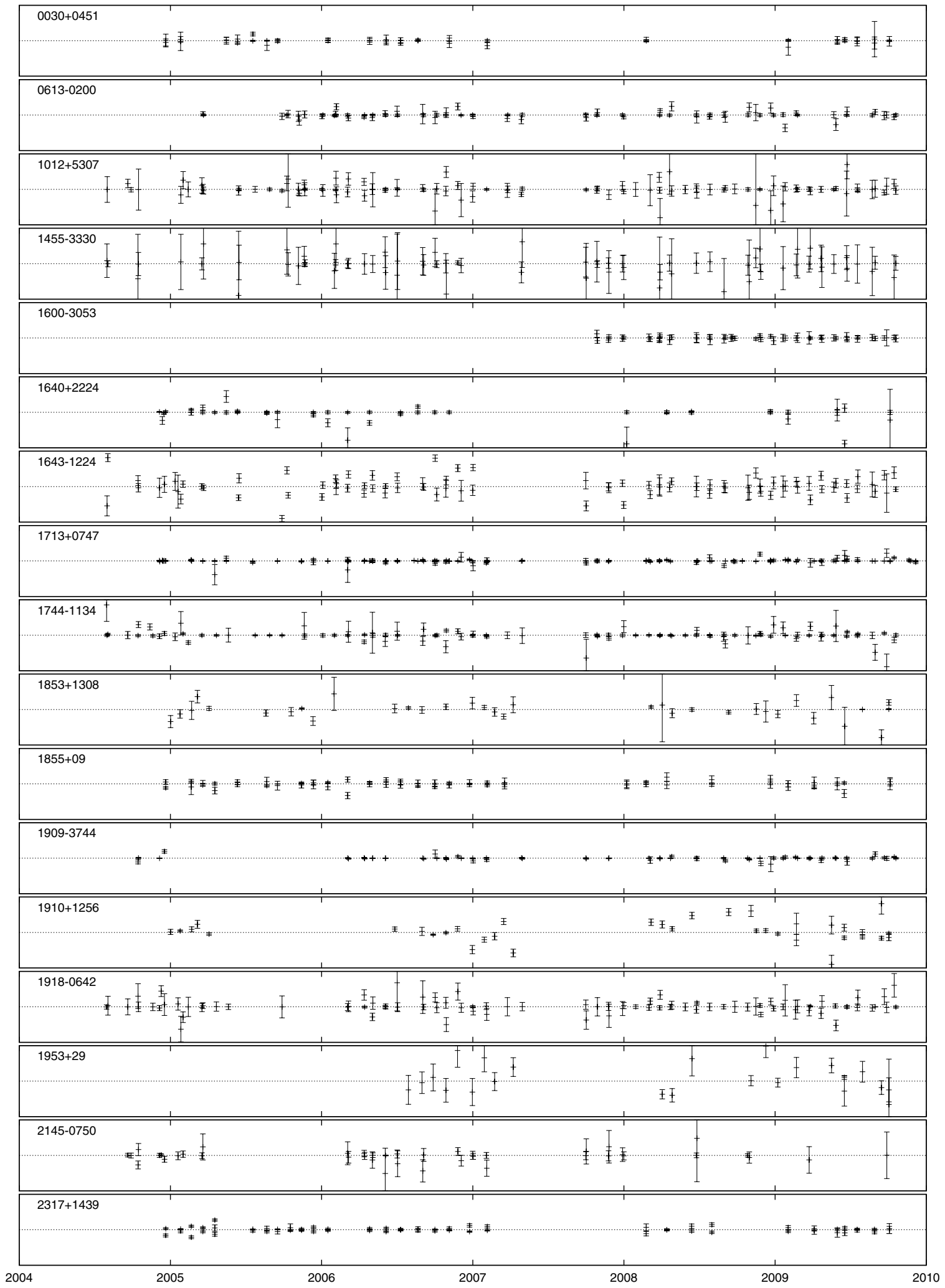

Figure 1. Overview of timing residuals for all sources, showing observational cadence and coverage during the five-year time span. The gap in 2007 was due to an extended maintenance period at both telescopes. The full scale of the $y$-axis is $10 \mu \mathrm{s}$ in all cases.

\subsection{Calibration and Time of Arrival Estimation}

As discussed in Section 2, the data products resulting from an observing session are a set of full-Stokes pulse profiles integrated over $4 \mathrm{MHz}$ radio bandwidth and one to three minutes of time, into either 2048 or 4096 pulse phase bins. Following standard pulsar data analysis procedures, we aim to determine from the profile data a set of pulse times of arrival (TOAs), i.e., times at which the apparent rotational phase of the pulsar passes through some fiducial point. This process involves several major steps: polarization calibration, template profile creation, additional profile averaging, and finally TOA measurement. For 
Table 1

List of Observed Millisecond Pulsars: Basic Parameters and Observing Setups

\begin{tabular}{|c|c|c|c|c|c|c|c|c|c|c|}
\hline \multirow[t]{2}{*}{ Source } & \multirow{2}{*}{$\begin{array}{c}P \\
(\mathrm{~ms})\end{array}$} & \multirow{2}{*}{$\begin{array}{l}d P / d t \\
\left(10^{-20}\right)\end{array}$} & \multirow{2}{*}{$\begin{array}{c}\mathrm{DM} \\
\left(\mathrm{pc} \mathrm{cm}^{-3}\right)\end{array}$} & \multirow{2}{*}{$\begin{array}{l}P_{b} \\
\text { (d) }\end{array}$} & \multicolumn{5}{|c|}{ Average Flux Density $(\mathrm{mJy})^{\mathrm{a}}$} & \multirow[t]{2}{*}{ Obs } \\
\hline & & & & & $(327 \mathrm{MHz})$ & $(430 \mathrm{MHz})$ & $(820 \mathrm{MHz})$ & $(1.4 \mathrm{GHz})$ & $(2.3 \mathrm{GHz})$ & \\
\hline $\mathrm{J} 0030+0451$ & 4.87 & 1.02 & 4.33 & $\ldots$ & $\ldots$ & 19.9 & $\ldots$ & 1.4 & $\ldots$ & $\mathrm{AO}$ \\
\hline J0613-0200 & 3.06 & 0.96 & 38.78 & 1.2 & $\ldots$ & $\cdots$ & 5.3 & 2.0 & $\cdots$ & GBT \\
\hline $\mathrm{J} 1012+5307$ & 5.26 & 1.71 & 9.02 & 0.6 & $\ldots$ & $\ldots$ & 7.6 & 3.9 & $\ldots$ & GBT \\
\hline $\mathrm{J} 1455-3330$ & 7.99 & 2.43 & 13.57 & 76.2 & $\ldots$ & $\ldots$ & 2.0 & 1.1 & $\cdots$ & GBT \\
\hline $\mathrm{J} 1600-3053$ & 3.60 & 0.95 & 52.33 & 14.3 & $\cdots$ & $\cdots$ & 3.1 & 2.3 & $\cdots$ & GBT \\
\hline $\mathrm{J} 1640+2224$ & 3.16 & 0.28 & 18.43 & 175.5 & $\ldots$ & 10.9 & $\ldots$ & 1.0 & $\ldots$ & $\mathrm{AO}$ \\
\hline J1643-1224 & 4.62 & 1.85 & 62.42 & 147.0 & $\ldots$ & $\ldots$ & 12.3 & 4.2 & $\ldots$ & GBT \\
\hline $\mathrm{J} 1713+0747$ & 4.57 & 0.85 & 15.99 & 67.8 & $\cdots$ & $\cdots$ & 8.8 & 6.3 & 3.6 & $\mathrm{AO}, \mathrm{GBT}$ \\
\hline J1744-1134 & 4.07 & 0.89 & 3.14 & $\ldots$ & $\ldots$ & $\ldots$ & 7.6 & 2.6 & $\ldots$ & GBT \\
\hline $\mathrm{J} 1853+1308$ & 4.09 & 0.87 & 30.57 & 115.7 & $\cdots$ & $\cdots$ & $\cdots$ & 0.2 & $\cdots$ & $\mathrm{AO}$ \\
\hline B1855+09 & 5.36 & 1.78 & 13.30 & 12.3 & $\cdots$ & 24.6 & $\cdots$ & 4.0 & $\cdots$ & $\mathrm{AO}$ \\
\hline J1909-3744 & 2.95 & 1.40 & 10.39 & 1.5 & $\ldots$ & $\ldots$ & 3.4 & 1.4 & $\ldots$ & GBT \\
\hline $\mathrm{J} 1910+1256$ & 4.98 & 0.97 & 34.48 & 58.5 & $\cdots$ & $\ldots$ & $\ldots$ & 0.2 & $\ldots$ & $\mathrm{AO}$ \\
\hline J1918-0642 & 7.65 & 2.57 & 26.60 & 10.9 & $\cdots$ & $\cdots$ & 4.5 & 1.8 & $\cdots$ & GBT \\
\hline B $1953+29$ & 6.13 & 2.97 & 104.50 & 117.3 & $\ldots$ & $\ldots$ & $\ldots$ & 1.0 & 0.1 & $\mathrm{AO}$ \\
\hline $\mathrm{J} 2145-0750$ & 16.05 & 2.98 & 9.03 & 6.8 & $\cdots$ & $\cdots$ & 12.3 & 3.2 & $\cdots$ & GBT \\
\hline $\mathrm{J} 2317+1439$ & 3.45 & 0.24 & 21.90 & 2.5 & 32.2 & 9.9 & $\cdots$ & $\cdots$ & $\cdots$ & $\mathrm{AO}$ \\
\hline
\end{tabular}

Note. ${ }^{\text {a }}$ The presence of flux density values indicate the frequencies at which each source is observed.

this work, we have performed all of these steps using two independent versions of pulsar data processing software, the first based on the PSRCHIVE ${ }^{20}$ software (Hotan et al. 2004), and the second based on ASPFitsReader (Ferdman 2008). This procedure provides an important cross-check for errors in the analysis software that might otherwise be hard to detect. Both analysis pipelines performed the same procedures, aside from template determination which was done only via PSRCHIVE.

Pulsar radio emission is typically highly polarized. While our current analysis relies only on the total intensity profiles for TOA determination, due to the high degree of polarization, calibration errors can still distort the intensity profile shape, leading to a TOA bias (e.g., van Straten 2006). A complete description of the instrumental response to a polarized signal is provided by the Mueller matrix, which is a radio-frequencydependent linear transformation from the intrinsic to observed Stokes parameters (Heiles et al. 2001; van Straten 2004). While we plan in future work to apply full Mueller matrix calibration to these data, for the current analysis we correct only the leading order terms, differential gain and phase between the two polarization components of the telescope signal. This is done via an injected calibration signal; immediately before or after each pulsar observation, a noise diode switched at $25 \mathrm{~Hz}$ is coupled into both polarization signal paths and measured with the pulsar backends. This provides a constant reference power versus frequency that is used to scale the pulsar data. The equivalent flux density of the calibration signal is determined separately for each polarization by observing the noise diode along with a bright, unpolarized quasar of known flux density (B1442+101 at Green Bank, J1413+1509 at Arecibo). This then provides a second scaling to convert the pulsar data to flux density units (Jy) separately in each linear (or circular for certain receivers) polarization. The two calibrated total power terms are then added together to form the total intensity ("Stokes $I$ ") profile, and the polarized profiles are not used further in this analysis.

The calibrated pulse profiles are used to determine pulse TOAs and their uncertainties by fitting for a pulse phase

20 http://psrchive.sourceforge.net shift between each profile and a standard "template" profile (Taylor 1992). The template ideally is a noise-free representation of the average pulse profile shape; any noise present in the template, especially if correlated with noise in the profiles, can bias the TOAs (Hotan et al. 2005). For this work we determine template profiles from our measured data in a twostep process: the profiles are first roughly aligned using a single-Gaussian template, then are summed together using weights to optimize the signal-to-noise ratio $(\mathrm{S} / \mathrm{N})$ in the final full-sum profile (see Demorest 2007, Equation (2.10)). We then apply a translation-invariant wavelet transform and thresholding (Coifman \& Donoho 1995) to the profile to remove its noise (Figure 2). The procedure is then iterated once, now using the new template for alignment, to produce the final template profiles used for determining TOAs. Before TOA determination, the template profiles are rotated so that the phase of their first harmonic component is zero. The wavelet noise removal procedure is implemented in the PSRCHIVE program psrsmooth. In this way, we obtained one template per pulsar per receiver used to observe it. While all other processing steps in this section were performed independently by both software pipelines, for consistency the same set of templates was used in both cases.

The standard template-matching procedure used for TOA determination in this analysis is known to suffer problems when applied to low-S/N data (Hotan et al. 2005). Therefore, it is useful to average profiles over as much time and radio bandwidth as possible to maximize the $\mathrm{S} / \mathrm{N}$ before forming TOAs, while still retaining enough resolution to measure all appropriate instrumental or astrophysical effects. In this analysis we will retain the native instrumental frequency resolution (see Section 3.2), and have chosen to average over time all profiles in a given frequency channel from each observing epoch (typically 30 minutes). TOAs are then measured for each average profile, resulting in a set of $\sim 20-30$ TOAs (one per $4 \mathrm{MHz}$ frequency channel) per each dual-receiver pair of observing epochs. The total number of TOAs for each pulsar are listed in Table 2. As previously mentioned, we computed TOAs using two independent analysis pipelines. After verifying that the two pipelines produced consistent results, we focus the remainder of 

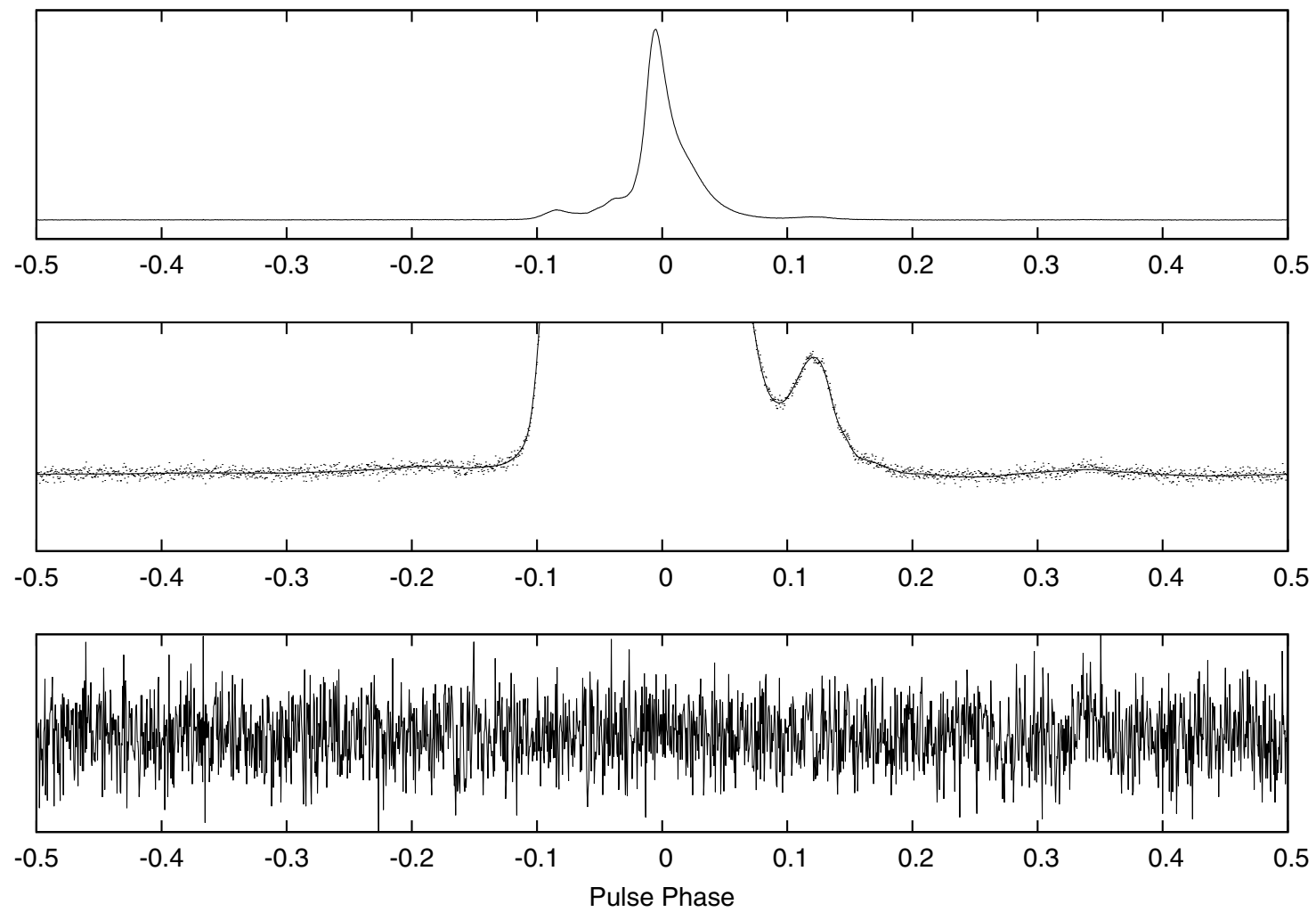

Figure 2. Full-sum profile and template profile for J1713+0747 at $1400 \mathrm{MHz}$. The top panel shows the full-sum profile at full scale. The middle panel shows the full-sum profile (points) and wavelet-denoised template version (line). The bottom panel shows the residual difference between the full-sum profile and template. The reference phase for TOA determination is at zero turns, in the center of the plot.

Table 2

Overview and Results from Timing Model Fits

\begin{tabular}{|c|c|c|c|c|c|c|c|c|c|c|}
\hline \multirow[t]{2}{*}{ Source } & \multirow{2}{*}{$\begin{array}{l}\text { No. of } \\
\text { TOAs }^{\mathrm{a}}\end{array}$} & \multicolumn{3}{|c|}{ No. of Parameters } & \multirow{2}{*}{$\begin{array}{l}\mathrm{rms} \\
(\mu \mathrm{s})\end{array}$} & \multirow[t]{2}{*}{ Fit $\chi^{2}$} & \multicolumn{3}{|c|}{ Epoch-averaged rms / median $\sigma_{t}(\mu \mathrm{s})^{\mathrm{c}}$} & \multirow[t]{2}{*}{ Figure Nos. } \\
\hline & & $\mathrm{DM}$ & Profile & Other $^{b}$ & & & Low-band ${ }^{\mathrm{d}}$ & High-band & Combined & \\
\hline $\mathrm{J} 0030+0451$ & 545 & 20 & 26 & 7 & 0.604 & 1.44 & $0.019 / 0.38$ & $0.328 / 0.35$ & $0.148 / 0.37$ & 4 \\
\hline J0613-0200 & 1113 & 34 & 45 & 12 & 0.781 & 1.21 & $0.021 / 0.17$ & $0.519 / 0.50$ & $0.178 / 0.30$ & 5 \\
\hline $\mathrm{J} 1012+5307$ & 1678 & 52 & 53 & 14 & 1.327 & 1.40 & $0.192 / 0.69$ & $0.345 / 0.65$ & $0.276 / 0.67$ & 6 \\
\hline J1455-3330 & 1100 & 37 & 53 & 12 & 4.010 & 1.01 & $0.363 / 1.66$ & $1.080 / 2.97$ & $0.787 / 2.35$ & 7 \\
\hline $\mathrm{J} 1600-3053$ & 625 & 21 & 31 & 14 & 1.293 & 1.45 & $0.233 / 0.52$ & $0.141 / 0.27$ & $0.163 / 0.34$ & 8 \\
\hline $\mathrm{J} 1640+2224$ & 631 & 23 & 26 & 12 & 0.562 & 4.36 & $0.057 / 0.20$ & $0.601 / 0.52$ & $0.409 / 0.22$ & 9 \\
\hline J1643-1224 & 1266 & 40 & 48 & 13 & 2.892 & 2.78 & $0.589 / 0.74$ & $1.880 / 0.62$ & $1.467 / 0.67$ & 10 \\
\hline $\mathrm{J} 1713+0747$ & 2368 & 50 & 111 & 15 & 0.106 & 1.48 & $0.092 / 0.14$ & $0.025 / 0.05$ & $0.030 / 0.08$ & 3 \\
\hline J1744-1134 & 1617 & 54 & 49 & 7 & 0.617 & 3.58 & $0.139 / 0.19$ & $0.229 / 0.26$ & $0.198 / 0.22$ & 11 \\
\hline $\mathrm{J} 1853+1308$ & 497 & 0 & 34 & 12 & 1.028 & 1.16 & $0.271 / 0.61$ & $0.096 / 1.38$ & $0.255 / 0.61$ & 12 \\
\hline B1855+09 & 702 & 29 & 21 & 14 & 0.395 & 2.19 & $0.277 / 0.49$ & $0.101 / 0.25$ & $0.111 / 0.41$ & 13 \\
\hline J1909-3744 & 1001 & 31 & 37 & 14 & 0.181 & 1.95 & $0.011 / 0.08$ & $0.047 / 0.15$ & $0.038 / 0.09$ & 14 \\
\hline $\mathrm{J} 1910+1256$ & 525 & 0 & 34 & 14 & 1.394 & 2.09 & $0.712 / 0.36$ & $0.684 / 0.89$ & $0.708 / 0.40$ & 15 \\
\hline J1918-0642 & 1306 & 49 & 37 & 12 & 1.271 & 1.21 & $0.129 / 0.52$ & $0.211 / 0.75$ & $0.203 / 0.62$ & 16 \\
\hline B1953+29 & 208 & 0 & 27 & 12 & 3.981 & 0.98 & $1.879 / 1.49$ & $0.543 / 3.33$ & $1.437 / 1.49$ & 17 \\
\hline $\mathrm{J} 2145-0750$ & 675 & 20 & 37 & 12 & 1.252 & 1.97 & $0.068 / 0.57$ & $0.494 / 0.81$ & $0.202 / 0.57$ & 18 \\
\hline $\mathrm{J} 2317+1439$ & 458 & 30 & 12 & 15 & 0.496 & 3.03 & $0.373 / 0.21$ & $0.150 / 0.18$ & $0.251 / 0.19$ & 19 \\
\hline
\end{tabular}

Notes.

a One TOA per frequency channel per epoch.

b "Other" parameters are all spin, astrometric and binary parameters as described in Section 3.2.

${ }^{c}$ Statistics computed from residuals averaged down to one point per receiver per epoch. See the text for details.

${ }^{d}$ Note that in these results, the low-frequency rms tends to be suppressed due to the $\mathrm{DM}(t)$ fit.

the analysis on the PSRCHIVE-produced data; all further results presented in this paper are specific to these data. These TOAs are the inputs for the next part of the analysis procedure, fitting the timing model. The entire set of TOAs used in this analysis can be obtained as an electronic supplement to this paper. ${ }^{21}$

\footnotetext{
21 http://www.cv.nrao.edu/ pdemores/nanograv_data
}

\subsection{Timing Model Fit}

The second part of the timing analysis is to fit the measured pulse TOAs for each pulsar to a physical timing model. The timing model predicts the apparent rotational phase of a pulsar based on a set of physical parameters describing the star's rotation (spin period, spin-down rate), astrometry (position, 
proper motion, parallax), binary orbital motion, and general relativistic effects such as Shapiro delay. The model prediction is compared to the measured TOAs and best-fit parameter values are determined via $\chi^{2}$ minimization. This procedure is a fundamental part of pulsar astronomy and has been described many times in the literature (see, for example, Lorimer \& Kramer 2004). For all results presented here, we use the standard TEMPO $^{22}$ timing analysis software. We have performed a detailed comparison between this analysis and one done on this data set using the newer TEMPO $2^{23}$ package (Hobbs et al. 2006), and obtained nearly identical results. For this data set, the rms difference between the TEMPO and TEMPO 2 residuals is typically $\sim 1 \mathrm{~ns}^{24}$ All results presented here used the JPL DE405 solar system ephemeris ${ }^{25}$ to determine positions of the Sun and Earth relative to the solar system barycenter.

As the main goal of this analysis is to detect or limit the $\mathrm{nHz}$ gravitational wave background, a detailed discussion of each pulsar's timing model parameters and the astrophysical significance of the results will not be presented here. The TEMPO parameter files containing the model parameters and fit results can be obtained along with the TOAs in the electronic supplement to the paper. However, the overall strategy for fitting the timing models can be described as follows:

1. The average pulsar spin frequency and frequency derivative (spin-down) were always fit parameters. No higher frequency derivatives were included.

2. All five astrometric terms - two sky coordinates, two components of proper motion, and parallax — were always fitted parameters.

3. Binary systems were fit for all five Keplerian parameters, using ether the "ELL1" or "DD" (Damour \& Deruelle 1985) timing models as appropriate. Additional relativistic or secular orbital terms were added only if the timing was significantly improved.

In addition to the model parameters just described, the timing fit also included terms to correct for time-variable DM and frequency dependence of the pulse profile shape. Dispersion is a propagation effect caused by the travel of the radio pulses through the ionized interstellar medium (ISM). This causes a radio frequency-dependent shift in pulse arrival time proportional to $\mathrm{DM} v^{-2}$. Astrophysically, DM represents the integrated column density of free electrons along the line of sight to the pulsar. Due to relative motion of the Earth and the pulsar, the effective path through the ISM changes with time, hence the DM varies in a stochastic manner (e.g., Ramchandran et al. 2006). For this experiment, we obtained (non-simultaneous) dual-frequency data as described in Section 2 specifically to measure and remove this effect from the timing. We have done this by including a piecewise-constant $\operatorname{DM}(t)$ function in the same fit that determines the other timing model parameters. For each observing epoch, a window of span up to 15 days is defined over which an independent DM offset is fit for. Epochs for which no dual-frequency data exist within a 15 day range were excluded from the analysis. This leads to a relative DM versus time measurement for each pulsar, as shown in Figure 3. The reference epoch for DM variation is the epoch of the first TOA in the lowest frequency channel. Due to variation in the observing bandwidth, this is not necessarily the first observing

\footnotetext{
22 http://tempo.sourceforge.net

$23 \mathrm{http}: / /$ tempo2.sourceforge.net

24 http://www.cv.nrao.edu/ pdemores/nanograv_data/tempo_compare.html

25 http://iau-comm4.jpl.nasa.gov/de405iom/
}

epoch. As previously noted, PSRs J1853+1308, J1910+1256 and B1953+29 have mostly single-band observations, so the DM variation has not been modeled for these three sources.

During the course of this analysis, we discovered additional radio frequency-dependent trends in pulse arrival times that were not well described by the $v^{-2}$ dispersion relation. We attribute these to the intrinsic evolution of the pulse profile shape with frequency (e.g., Kramer et al. 1999). The interstellar medium is another possible cause; however, since the effect does not appear to be time-variable an ISM explanation seems less likely. Regardless of its physical origin, any profile shape change versus frequency can lead to systematic TOA biases as follows: Although we have used separate template profiles per receiver, within each receiver band the template profile is constant. If the true profile shape is changing versus frequency, this will cause small but measurable systematic effects. To correct for these biases, we have included as free parameters in the timing fit a constant (in time) offset—also known as a "jump"-for each $4 \mathrm{MHz}$ frequency channel. These constant-in-time perchannel offsets are completely covariant with a constant-in-time dispersion measure, therefore this analysis can not determine both parameters uniquely. However, DM variations in the data set as described above can still be determined.

A summary of the timing analysis and results is presented in Table 2. For each pulsar, the total number of TOAs, and the total number of fit parameters is given. These are divided into those relating to the DM variation, frequency-dependent terms, and the other standard spin, astrometric and binary parameters. The success of the timing model fit can be characterized by analyzing the post-fit residuals, i.e., the difference between the observed and model-predicted arrival times. The uncertainty-weighted rms residual value and normalized $\chi^{2}$ values as determined directly from the fit are listed in the table. The majority of $\chi^{2}$ values fall near 1 , but are as high as $\sim 4$ in some cases. These values have been computed without the use of any multiplicative or additive modifications to the TOA uncertainties. ${ }^{26}$

Traditionally, pulsar timing results have been presented using TOAs that come from an average of all data taken during a given day. Here, our fit procedure requires the multi-frequency data be kept separate, so we cannot perform this averaging prefit. To facilitate comparison with previous work, the columns in Table 2 under the "epoch-averaged" heading give statistical properties of the residuals after first averaging the raw post-fit residuals down to one point per receiver per epoch. Weighted rms and median uncertainty are then computed from the epochaveraged residuals. The epoch-averaged statistics of the lowfrequency and high-frequency bands for each pulsar are shown separately as well as the combined value using all the data. Due to the $\mathrm{DM}(t)$ fit, the low-frequency rms tends to be suppressed, making it less useful as a simple characterization of the timing. This effect can be illustrated by comparing the low-frequency rms with the median uncertainty-in most cases, the median uncertainty is at least a factor of several larger than the rms. However, the high-frequency rms is not affected in the same way, and is generally comparable with the median uncertainty.

The two best pulsars in the set, PSRs J1713+0747 and J1909-3744, both have epoch-averaged rms in the $~ 30-50 \mathrm{~ns}$ range with comparable values for both the high-frequency and combined versions of this statistic. Timing residuals, in both raw and daily averaged forms, along with the measured $\mathrm{DM}(t)$ values

\footnotetext{
26 The "EFAC" and "EQUAD" options, respectively, in TEMPO. These parameters were sometimes used in previous timing analyses to compensate for unexplained systematic errors in TOAs or their uncertainties.
} 

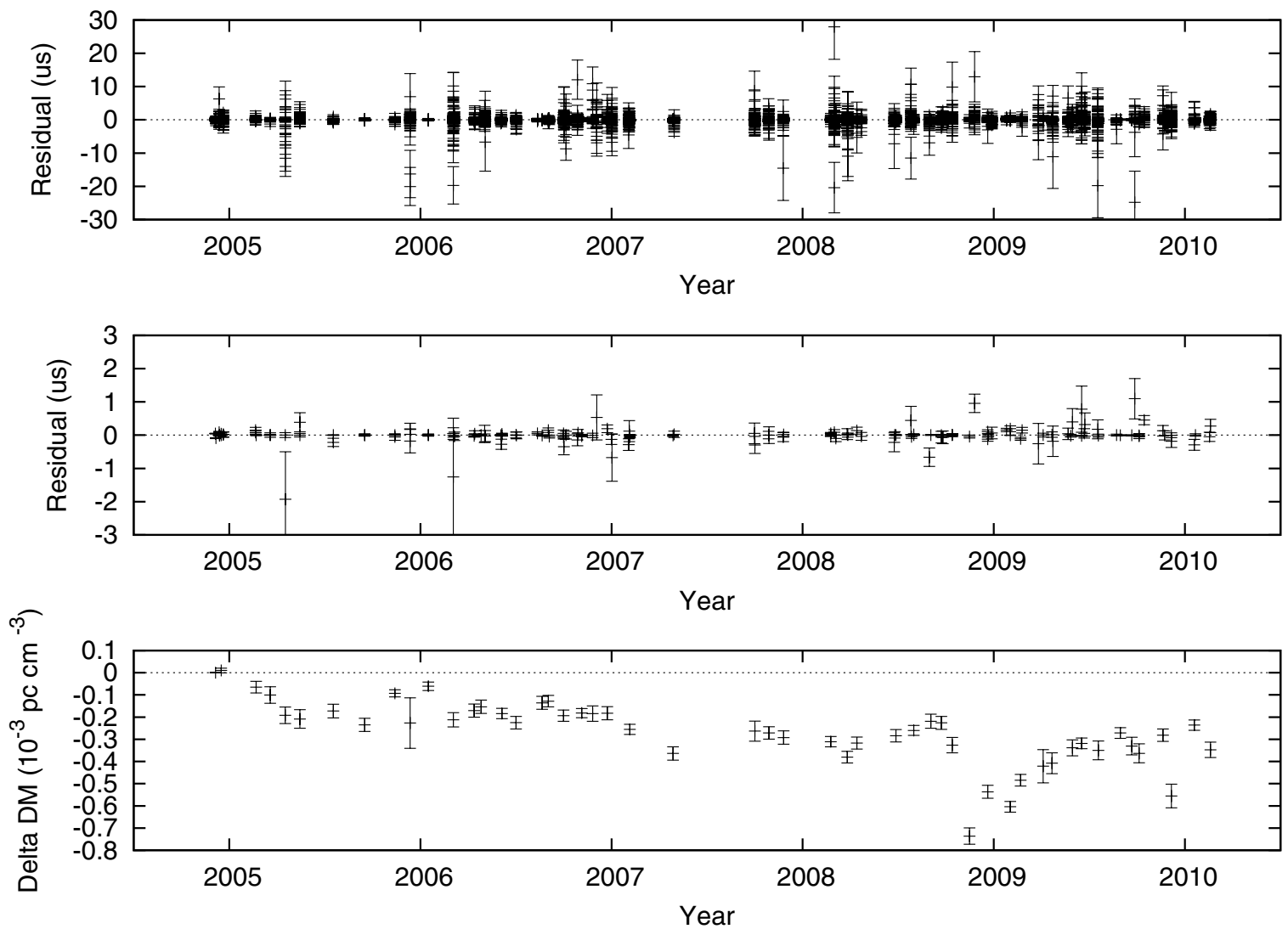

Figure 3. Timing summary for PSR J1713+0747. The top panel shows residuals from the multi-frequency TOAs used in the timing fit. The middle panel shows the same residuals averaged down to one point per band per day. The bottom panel shows the measured variation in DM as a function of time.

are shown in Figure 3 for PSR J1713+0747, and in Figures 4-19 for the other sources. The figure number for each pulsar's data is also listed in the final column of Table 2.

\section{GRAVITATIONAL WAVE ANALYSIS}

The presence of gravitational waves (GW) along the line-ofsight from a pulsar to Earth alters the effective path length in a time-variable manner, resulting in extra perturbations in pulsar timing residuals (Detweiler 1979). The observed amplitude of a single pulsar's timing residuals can therefore be used to limit the strength of any GW that may exist (Kaspi et al. 1994; Jenet et al. 2006). However, as an observed timing perturbation could also arise from a number of non-GW sources, this method can not be used to definitively confirm the presence of GW. It was first noted by Hellings \& Downs (1983) that a GW signal induces correlated variations in the timing residuals of a set of pulsars. The form of this correlation is unique to GW among the expected perturbations, and attempting to detect it is the basis for most current PTA efforts (van Haasteren et al. 2011; Yardley et al. 2011), including this work.

In this analysis, we will search for a stochastic gravitational wave background (GWB) signal in the pulsar timing results presented in Section 3. We assume the GWB will take the form which has become standard in this field-a power-law frequency spectrum and isotropic angular distribution. This signal is expected to be generated by the sum of unresolved SMBH binary systems with masses of $\sim 10^{8} M_{\odot}$ and orbital periods of 1-10 years. In this case, the characteristic strain spectrum is expected to have a "red" power-law spectral index $\alpha=-2 / 3$ (e.g., Jaffe \& Backer 2003; Sesana et al. 2008), with a possible break near $10 \mathrm{nHz}$ due to the finite number of sources (Sesana et al. 2008). A GWB of this form could also be generated from cosmic superstrings, with $\alpha=-7 / 6$ (Damour \& Vilenkin 2005; Siemens et al. 2007), or as inflationary "relics" with spectral index $\alpha=-1$ (Grishchuk 2005). Any GW signal will produces correlation in the timing fluctuations of pairs of pulsars. In the specific case of an isotropic GWB, the amount of correlated power is a function only of the angular separation of the two pulsars in the pair, and has a characteristic functional form first predicted by Hellings \& Downs (1983).

In this paper, we adopt definitions of the expected gravitational wave spectrum and its effect on timing consistent with previous papers on the topic (e.g., Jenet et al. 2006; van Haasteren et al. 2011). In particular, we assume a power-law spectrum in characteristic strain:

$$
h_{c}(f)=A_{f_{0}}\left(\frac{f}{f_{0}}\right)^{\alpha} .
$$

Here, $A_{f_{0}}$ is the unknown GW spectrum amplitude at a reference frequency $f_{0}$. For consistency with previous literature, we set $f_{0}=1 \mathrm{yr}^{-1}$, and will call the resulting amplitude $A_{1}$. This GWB produces a fluctuation $y(t)$ in the pulse times of arrival from a given pulsar, with power spectrum given by

$$
S_{y}(f)=\frac{1}{12 \pi^{2}} \frac{1}{f^{3}} h_{c}(f)^{2} .
$$

It is important to note that $y(t)$ represents the pre-fit contribution of the GW signal to the pulse TOAs. The effect of the timing model fit will be considered in Section 4.1. Also important is that this formulation of $S_{y}(f)$ is consistent with that used by 

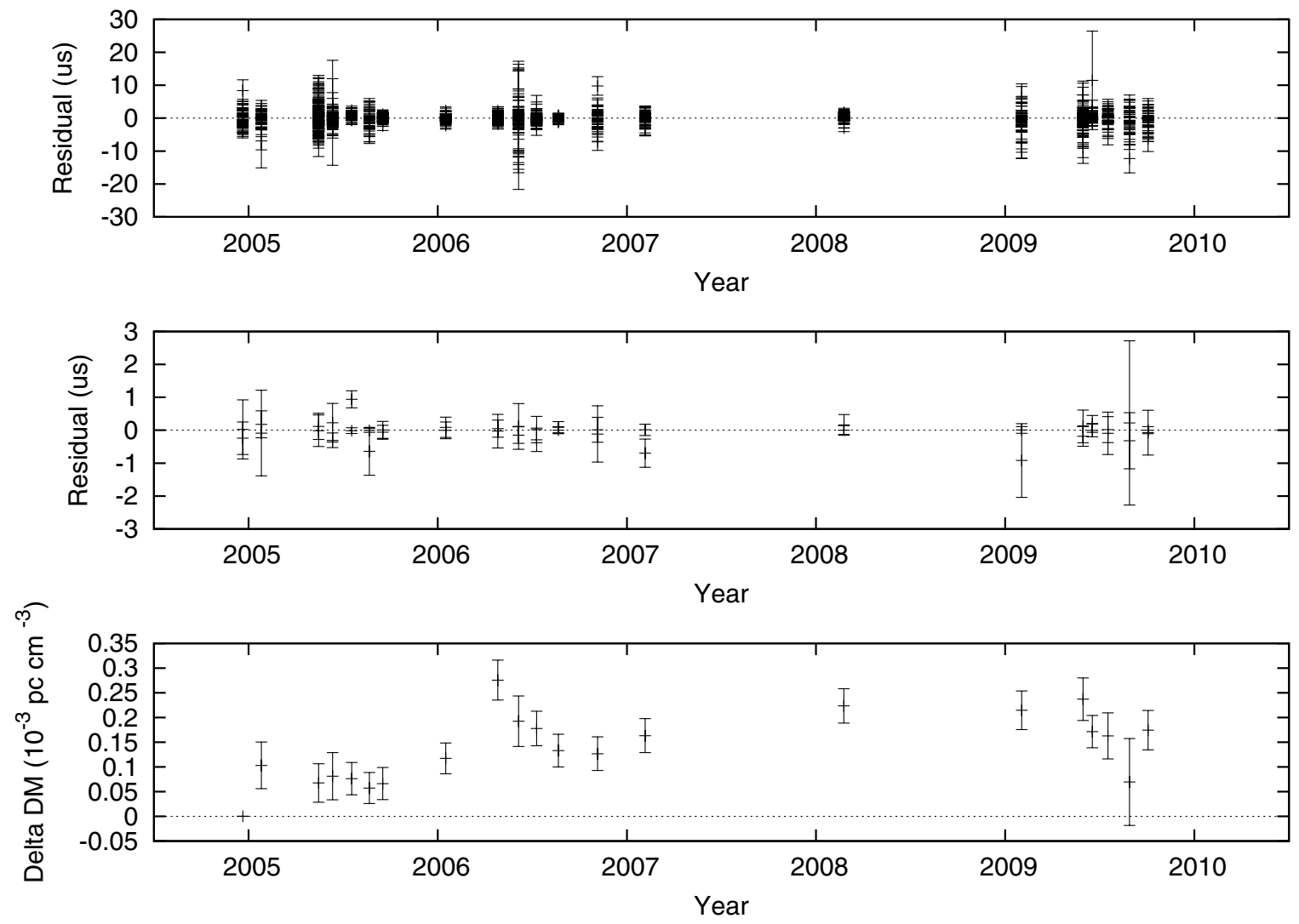

Figure 4. Timing summary for PSR J0030+0451, see Figure 3 for details.
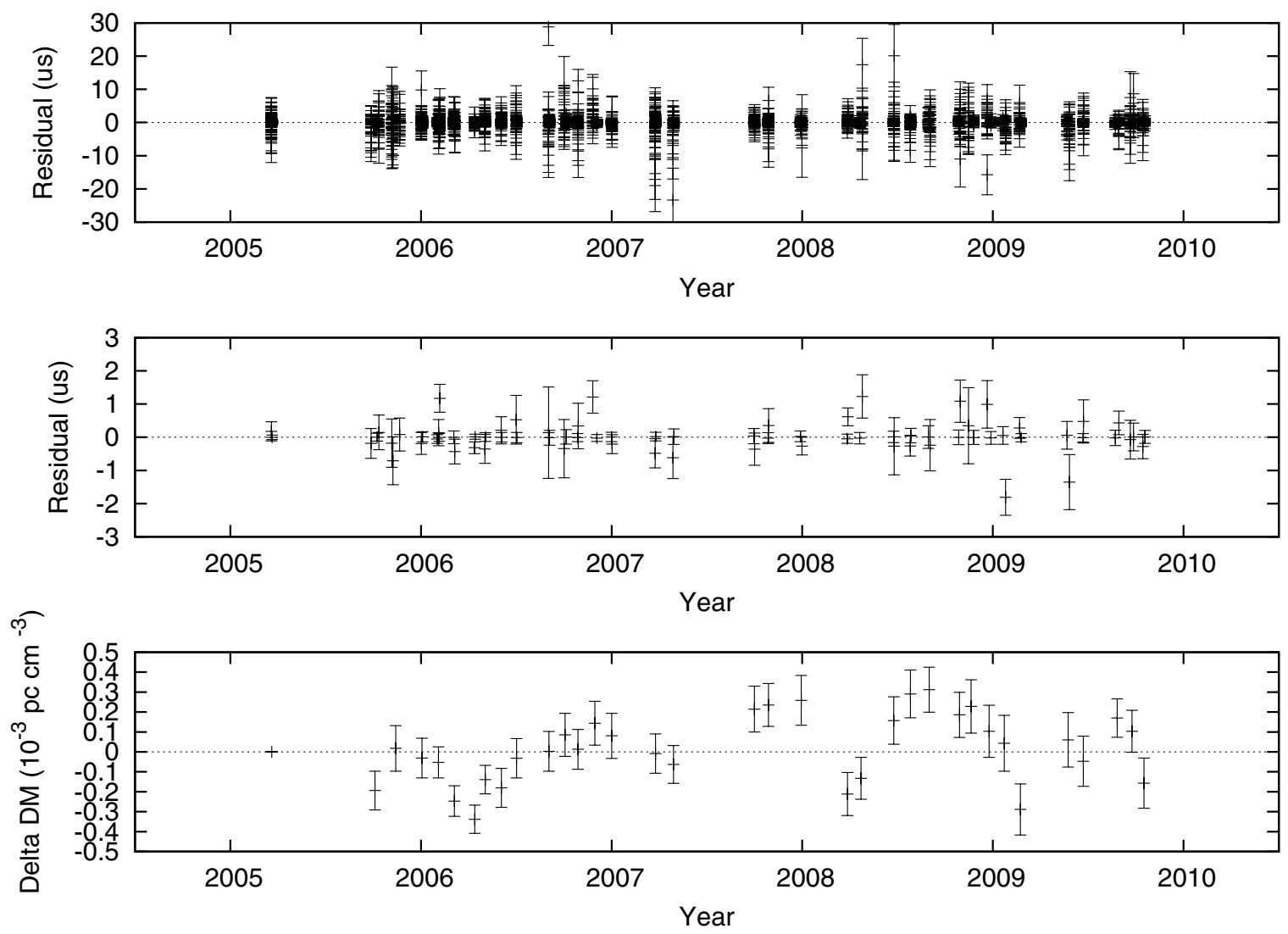

Figure 5. Timing summary for PSR J0613-0200, see Figure 3 for details. 

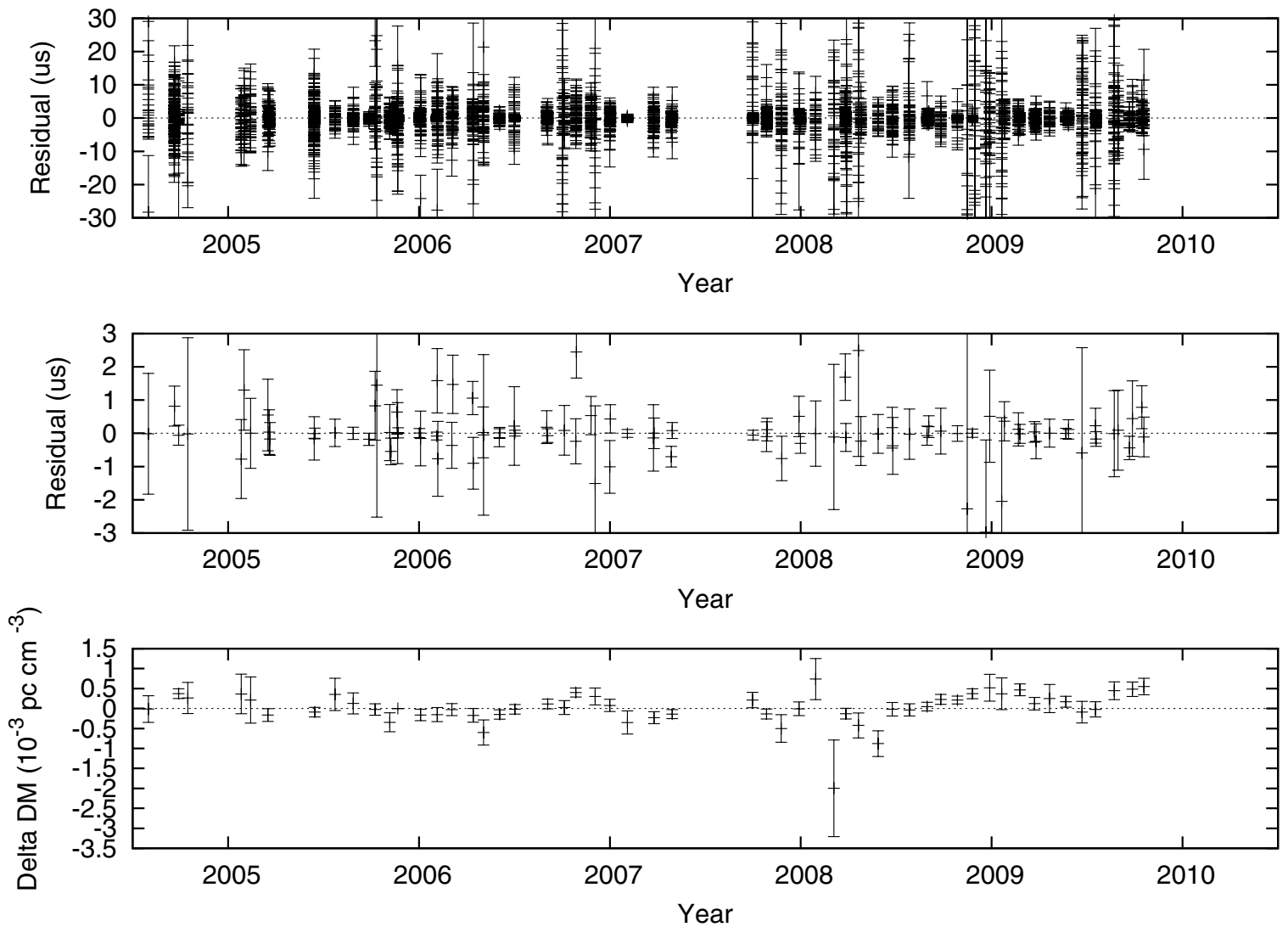

Figure 6. Timing summary for PSR J1012+5307, see Figure 3 for details.
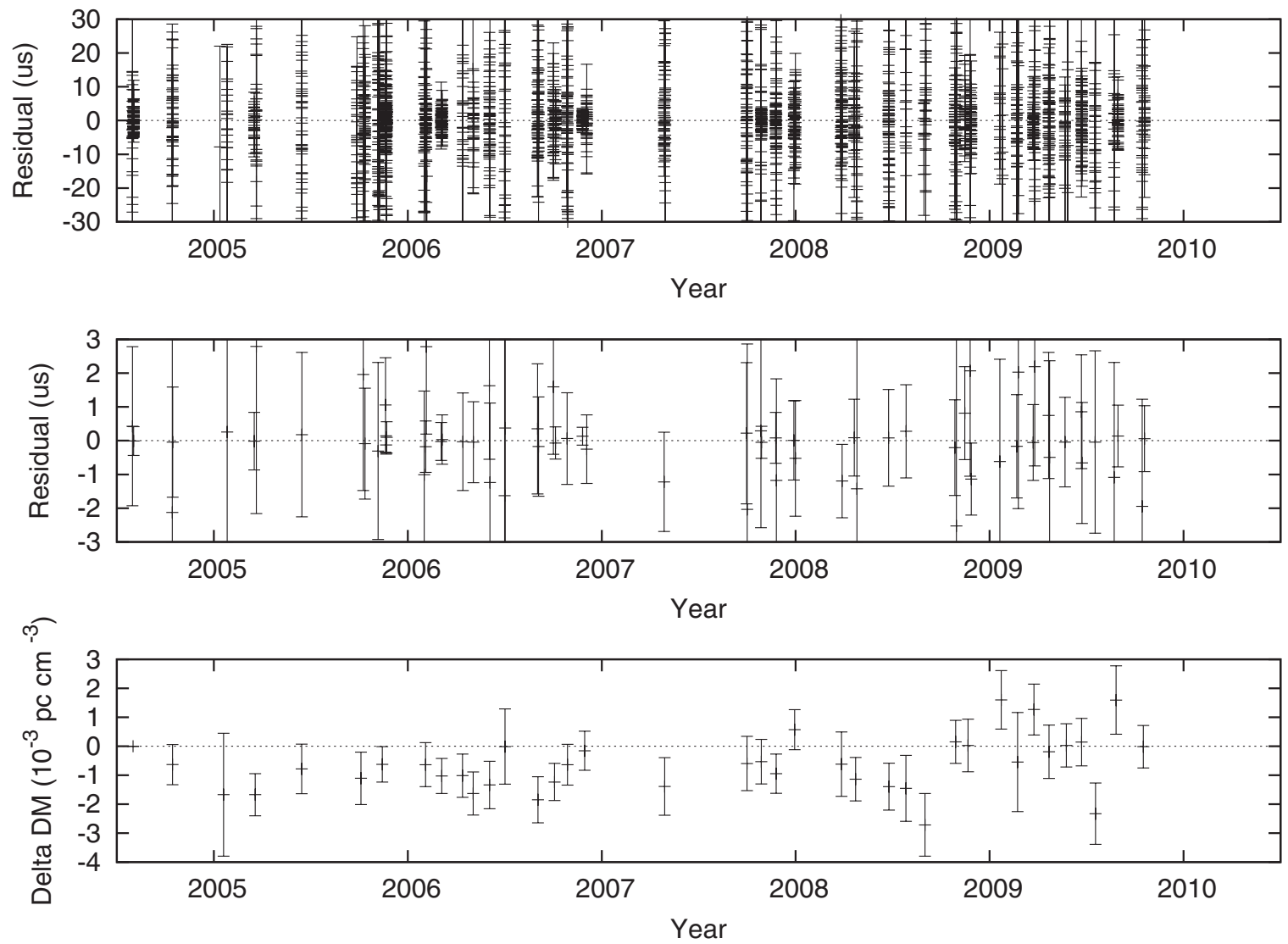

Figure 7. Timing summary for PSR J1455-3330, see Figure 3 for details. 

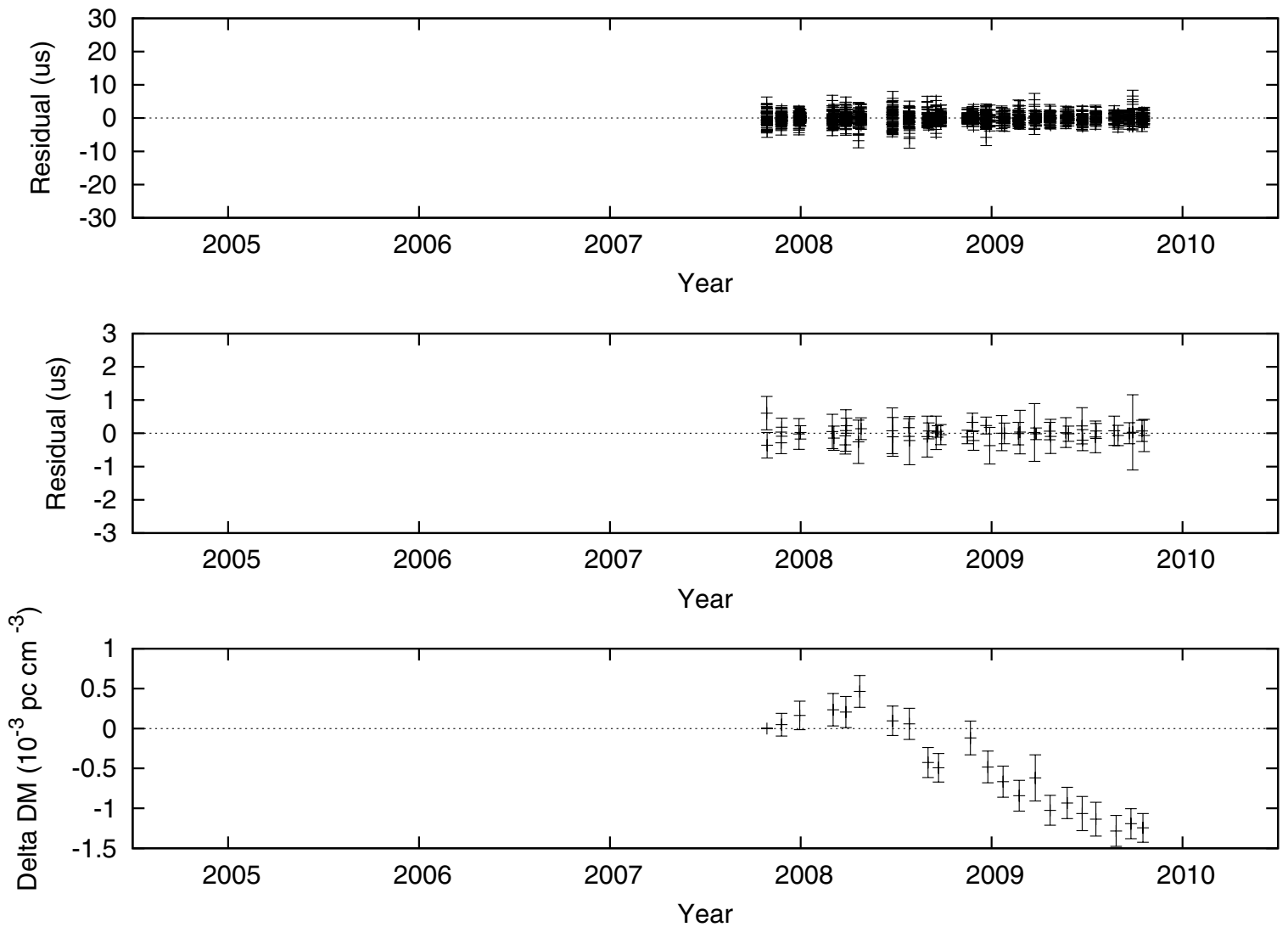

Figure 8. Timing summary for PSR J1600-3053, see Figure 3 for details.
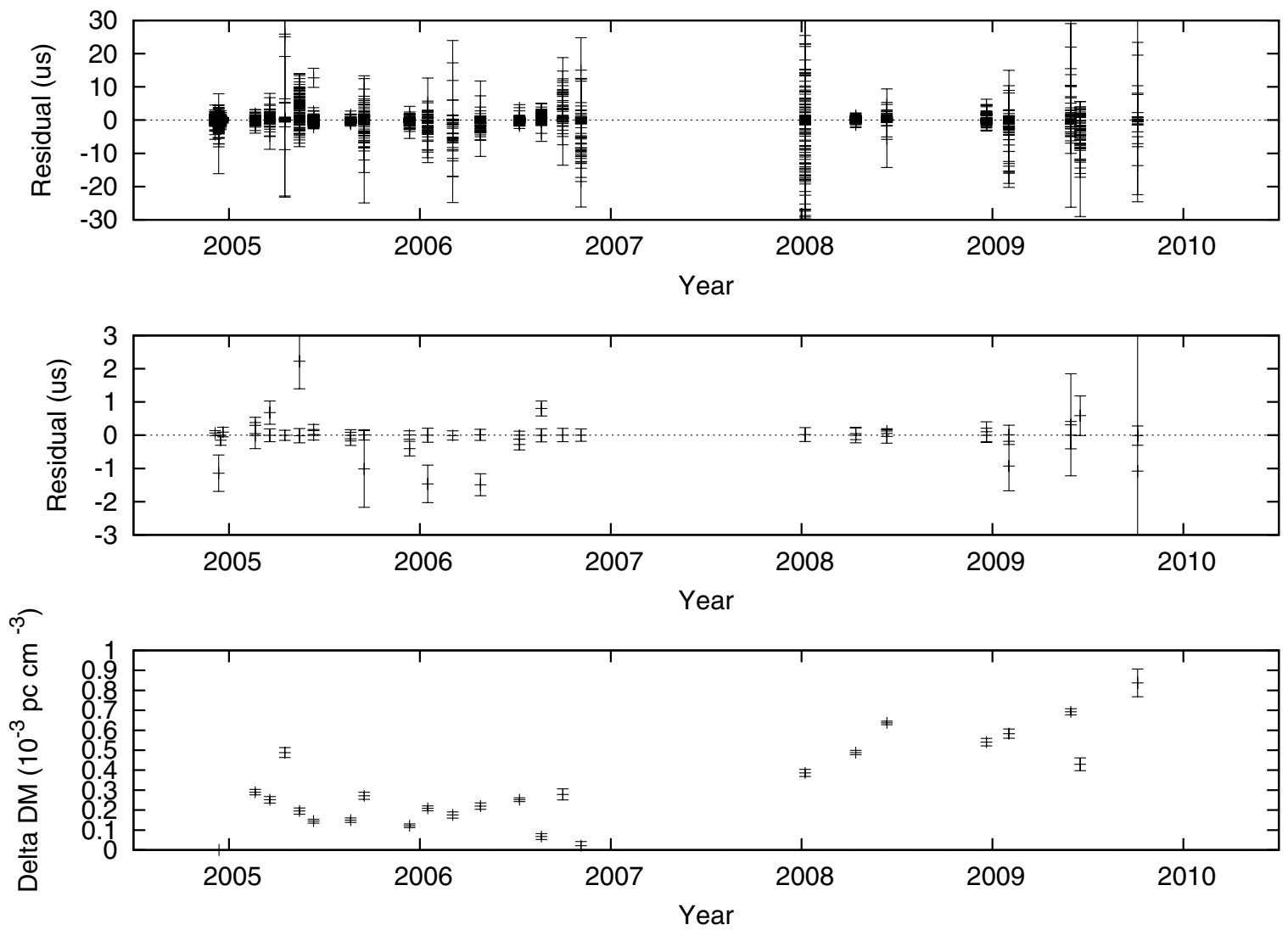

Figure 9. Timing summary for PSR J1640+2224, see Figure 3 for details. 

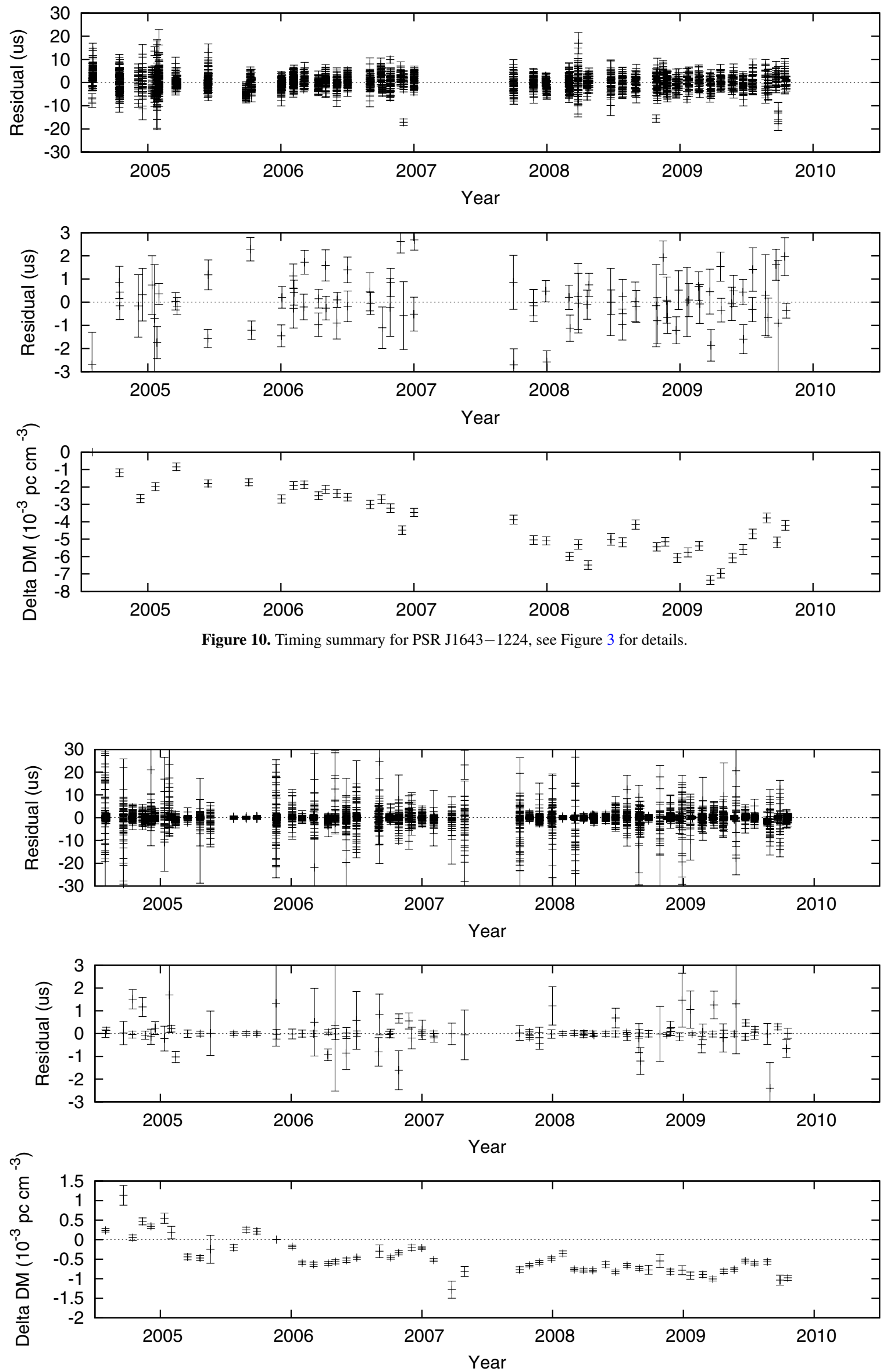

Figure 11. Timing summary for PSR J1744-1134, see Figure 3 for details. 

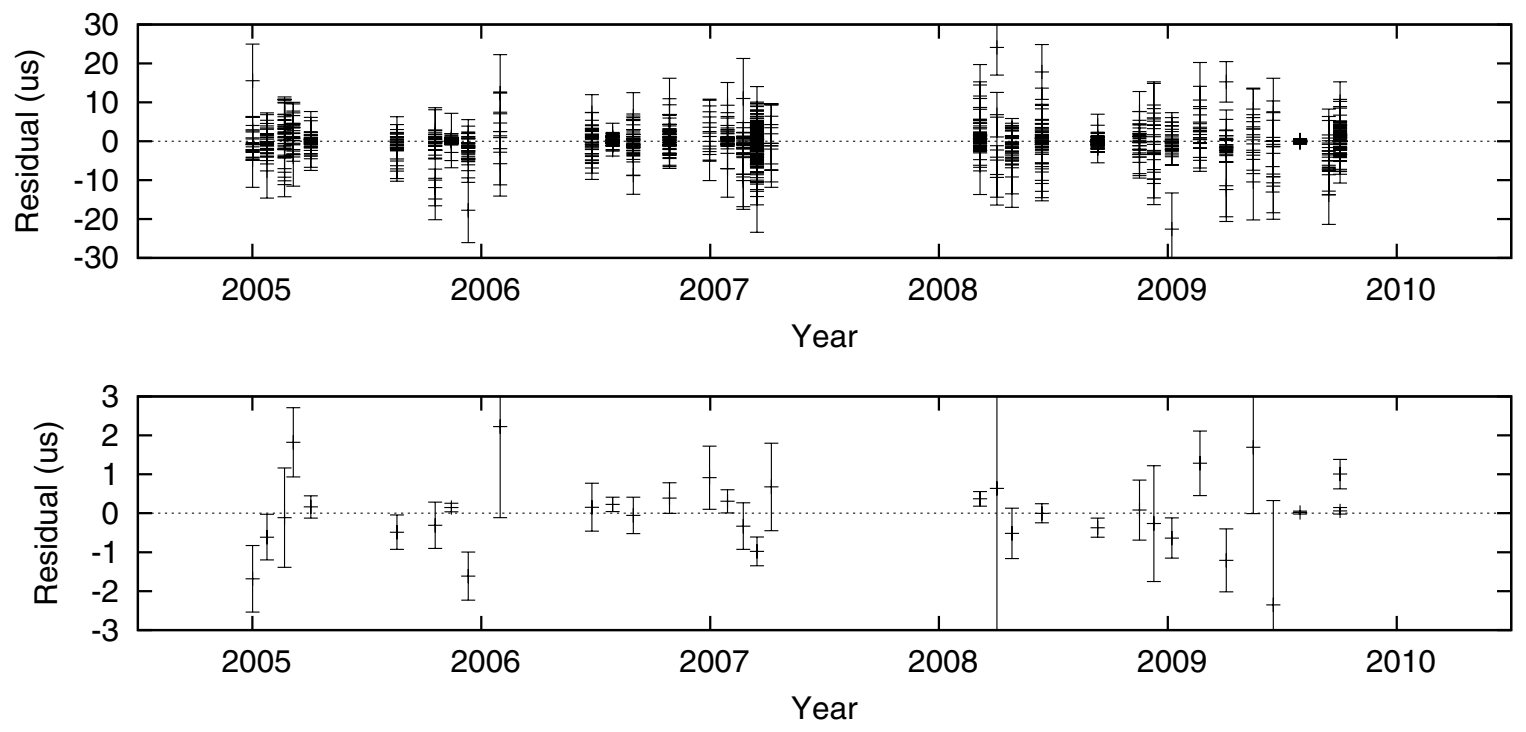

Figure 12. Timing summary for PSR J1853+1308, see Figure 3 for details.
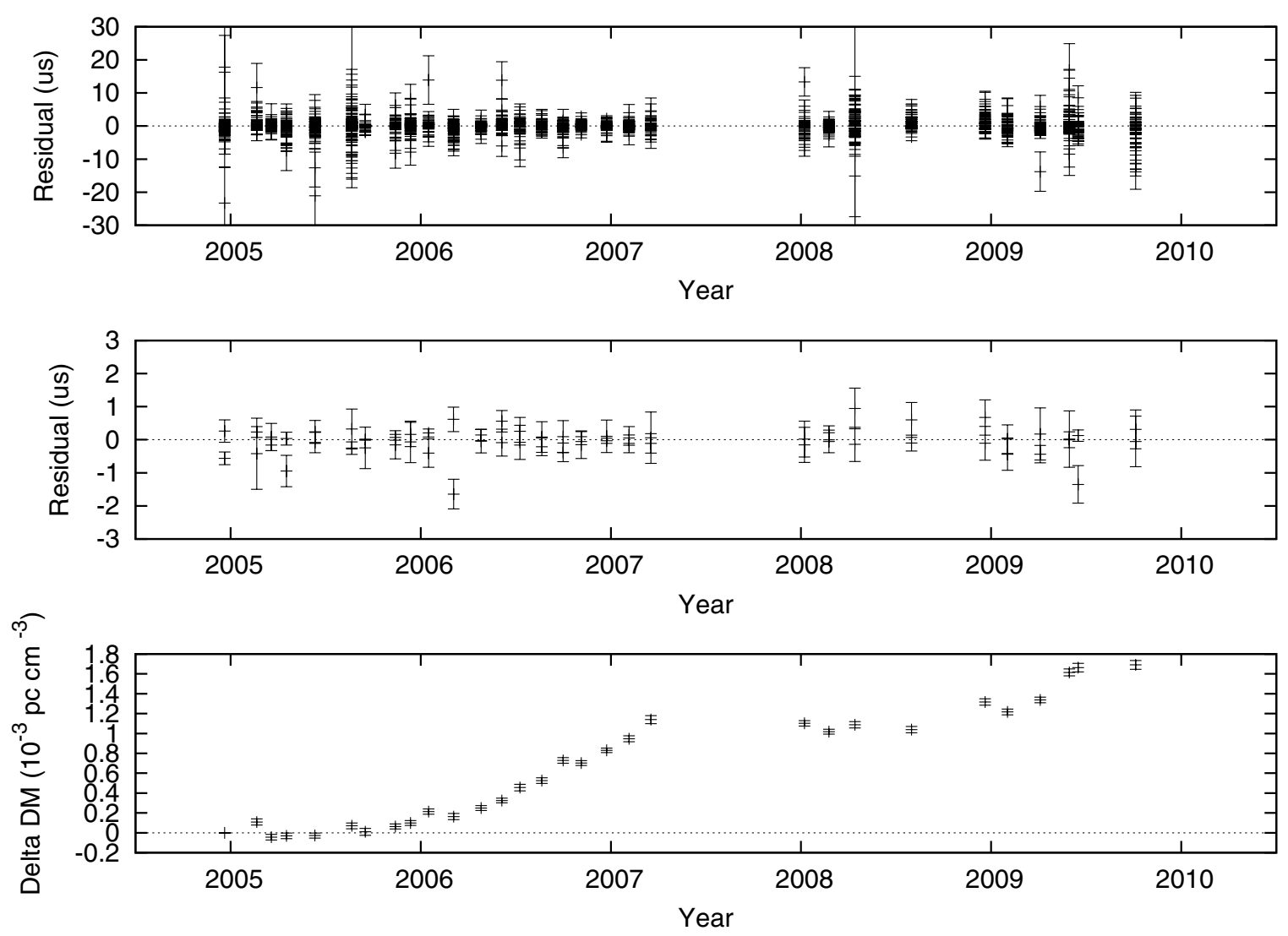

Figure 13. Timing summary for PSR B $1855+09$, see Figure 3 for details.

Jenet et al. (2006), Hobbs et al. (2009), and van Haasteren et al. $(2011)^{27}$ but is a factor of three smaller than that used by Jenet et al. (2005). This results in a factor of $\sqrt{3}$ difference in limits on $A_{1}$ depending on which definition is in use, and care

27 While the equations given by van Haasteren et al. (2011) used the Jenet et al. (2005) definition, their published limit of $A_{1}<6 \times 10^{-15}$ was computed using the same scaling as our Equation (2) (R. van Haasteren 2012, private communication). should therefore be taken when directly comparing the various published limits.

It is useful to compute the expected time-domain correlation between pairs of timing fluctuations from pulsars $a$ and $b$ :

$$
C_{y, i j}^{(a b)}=E\left\{y_{a}\left(t_{i}\right) y_{b}\left(t_{j}\right)\right\}=C_{y}\left(t_{i}-t_{j}\right) \zeta\left(\theta_{a b}\right) .
$$

Here, $\theta_{a b}$ is the angular separation between pulsars $a$ and $b$, and $\zeta\left(\theta_{a b}\right)$ is the Hellings-Downs function describing the 

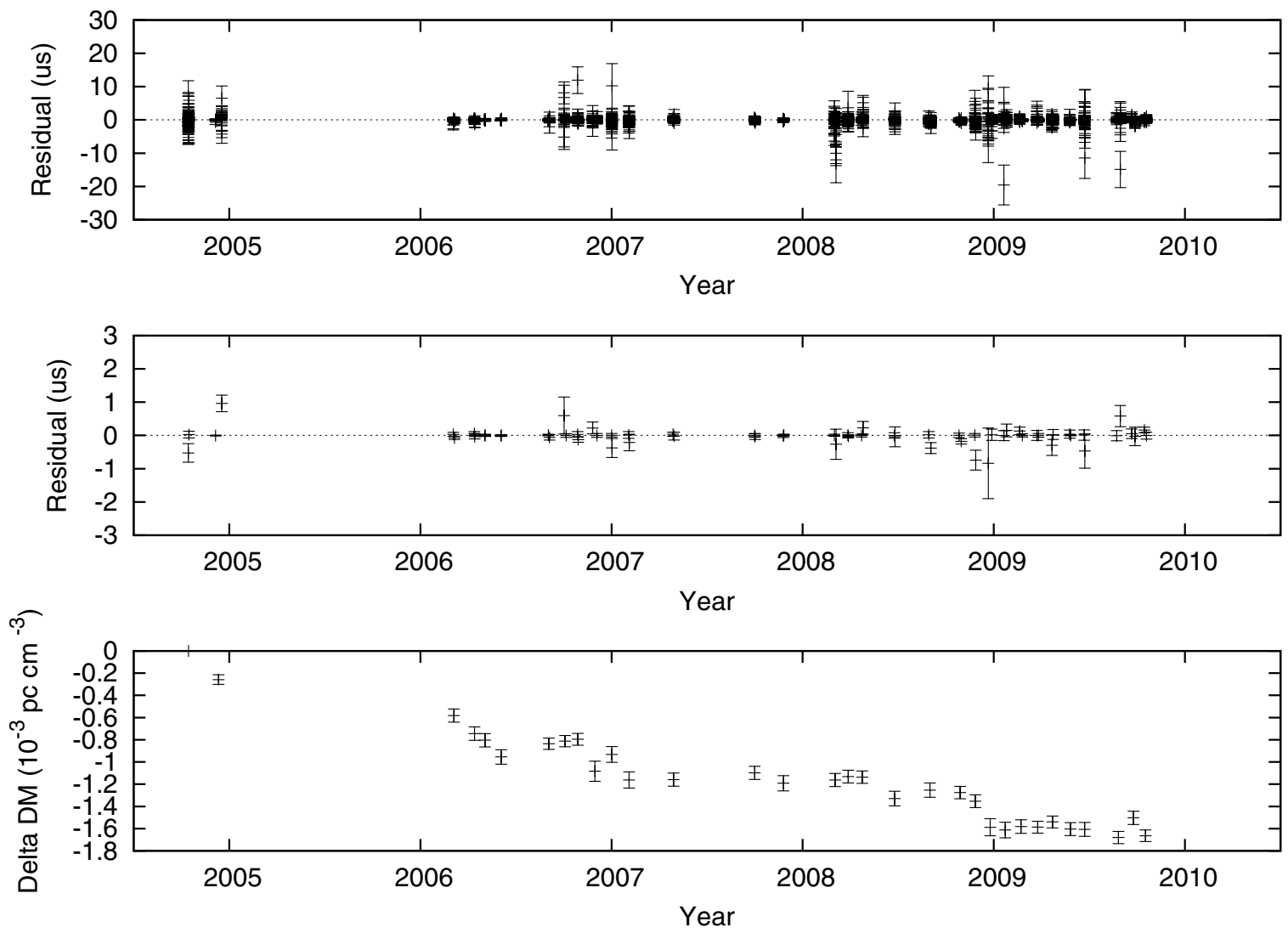

Figure 14. Timing summary for PSR J1909-3744, see Figure 3 for details.
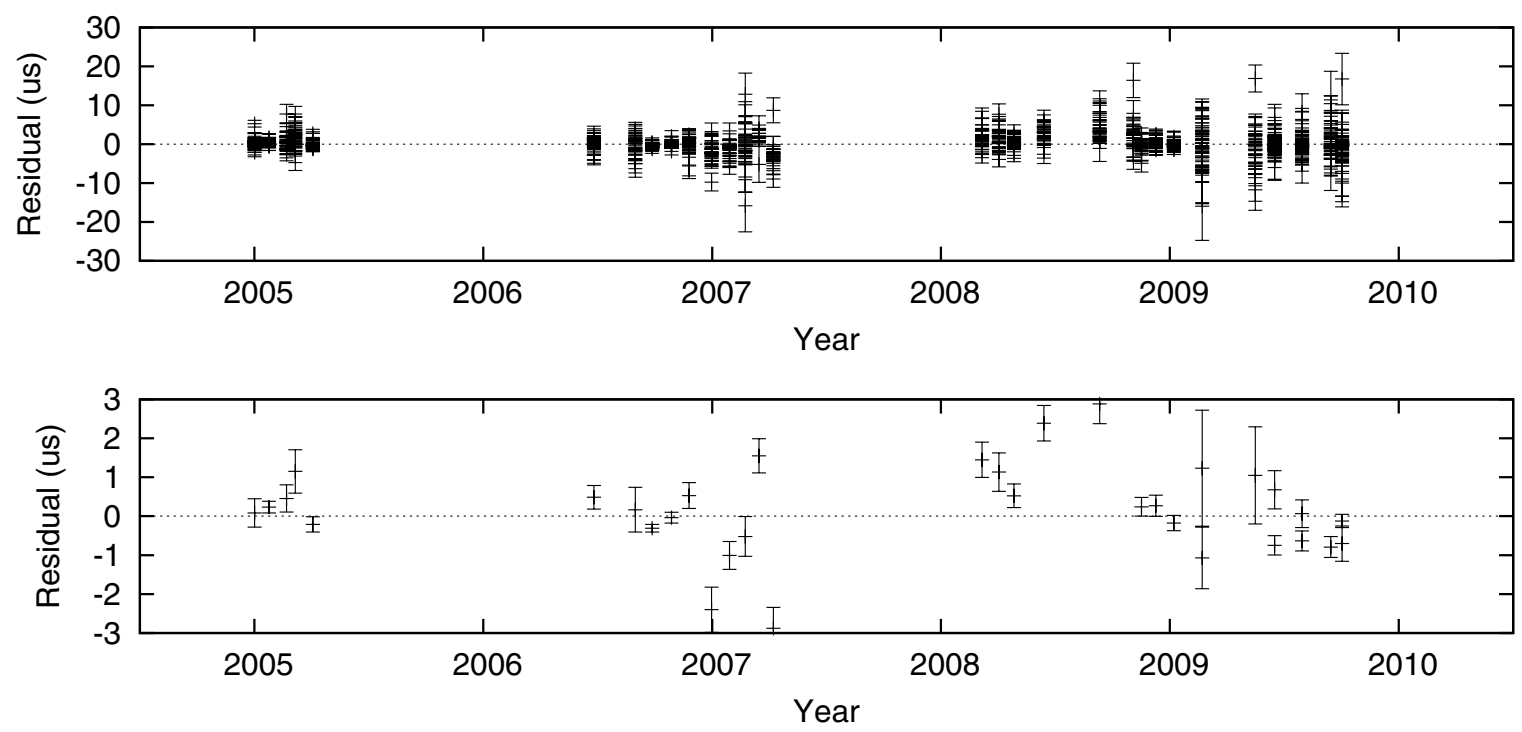

Figure 15. Timing summary for PSR J1910+1256, see Figure 3 for details.

expected angular correlation for a isotropic GWB. $C_{y}(\tau)$ is the GW signal autocorrelation (Fourier transform of $S_{y}(f)$ ), and can be computed analytically for a power-law spectrum as presented by van Haasteren et al. (2009, 2011). Additionally, here and in the following, $E\{\cdot\}$ represents the statistical expectation value, or average over many realizations of the enclosed quantity. This description assumes the GW signal follows wide-sense stationary statistics (e.g., Papoulis \& Pillai 2002).

In the following sections we first present methodology for analyzing the timing residuals from Section 3, and discuss the effect that fitting the timing model has on the statistics of post-fit residuals. We will then apply these methods to first determine an upper limit to the stochastic GWB amplitude using a single 

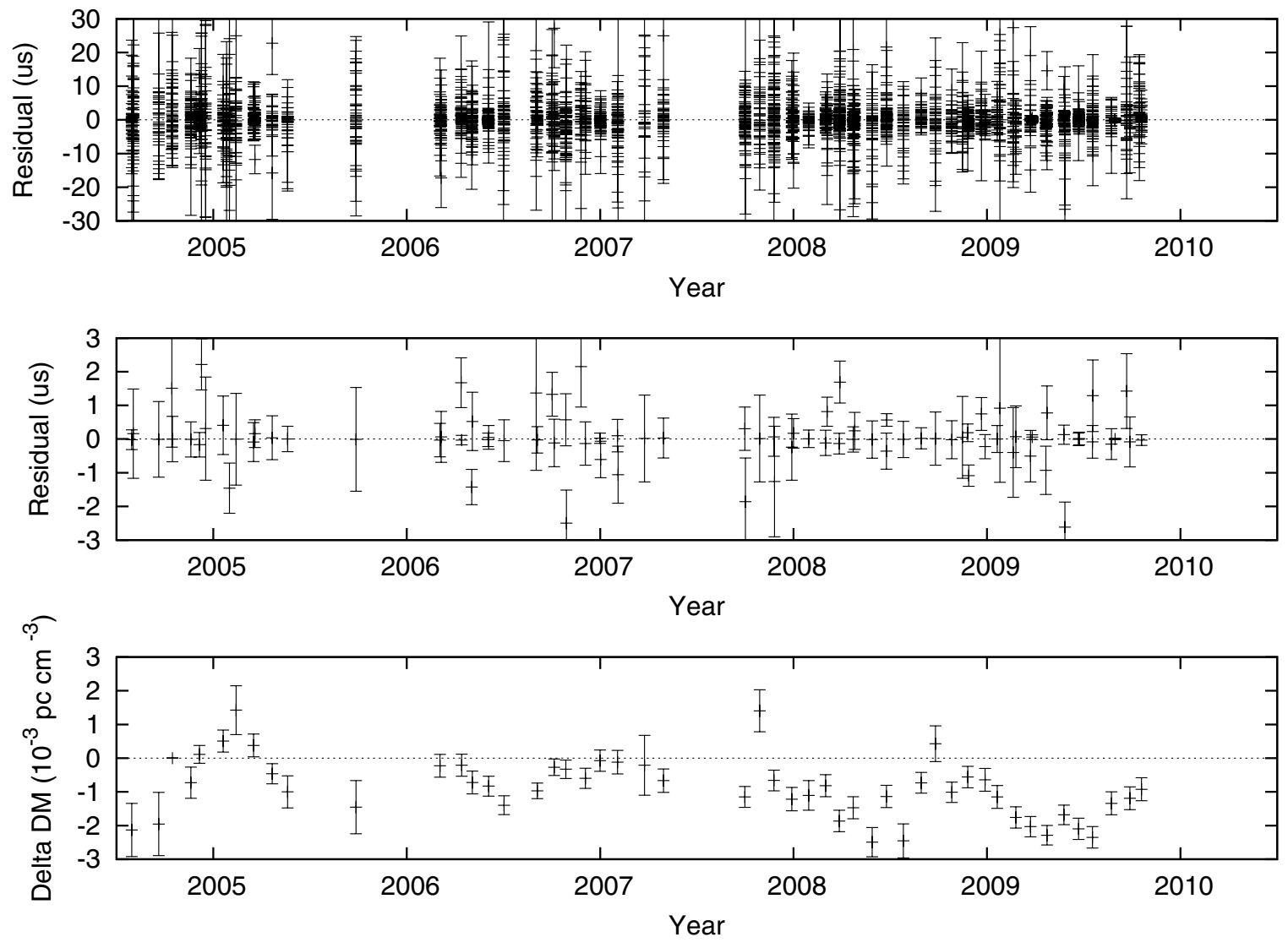

Figure 16. Timing summary for PSR J1918-0642, see Figure 3 for details.
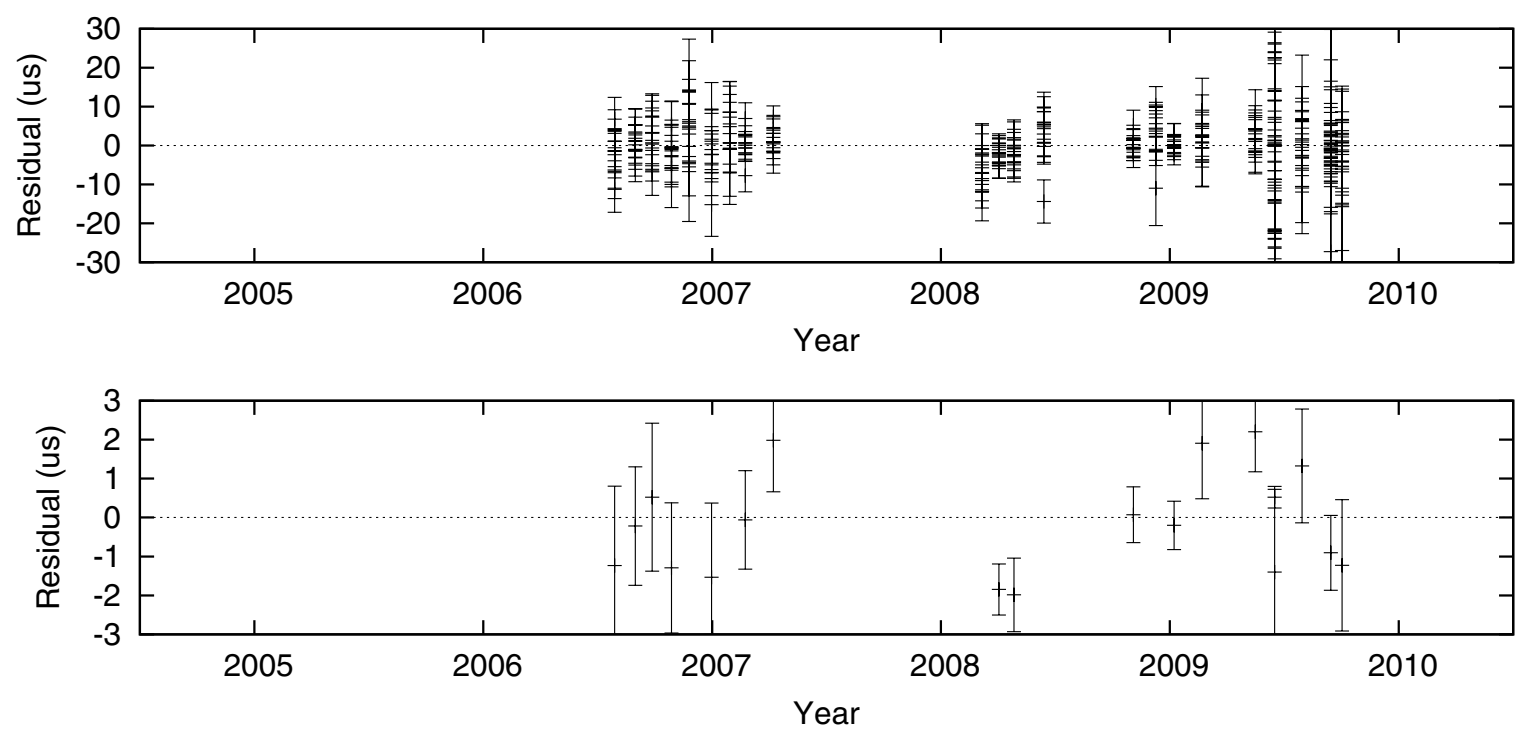

Figure 17. Timing summary for PSR B1953+29, see Figure 3 for details.

pulsar, and then attempt to detect or limit the GWB by looking for angular correlations.

\subsection{Effect of the Timing Model Fit}

In order to use pulsar timing data to detect gravitational waves, one must account for the fact that the pulsar parameters (spin period, astrometric parameters, etc.) are not known a priori, and need to be determined from the same data that are used for $\mathrm{GW}$ detection. Especially in the case of red GW spectra, a large fraction of the GW signal power is covariant with the "longterm" pulsar parameters such as spin period and spin-down rate, and cannot be unambiguously separated from these intrinsic pulsar features. Previous analyses have typically dealt with this by decomposing timing residuals using a set of polynomials 

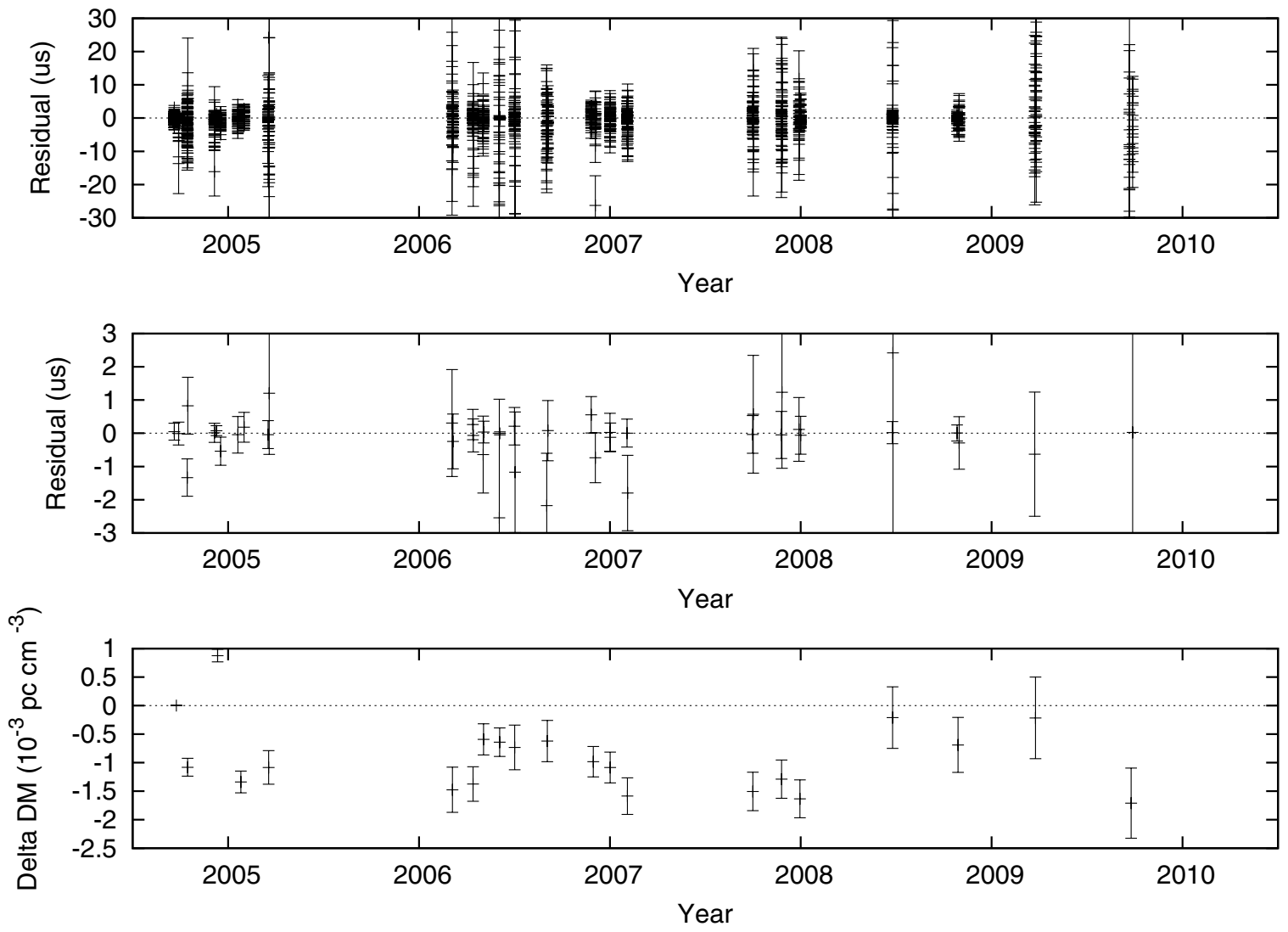

Figure 18. Timing summary for PSR J2145-0750, see Figure 3 for details.
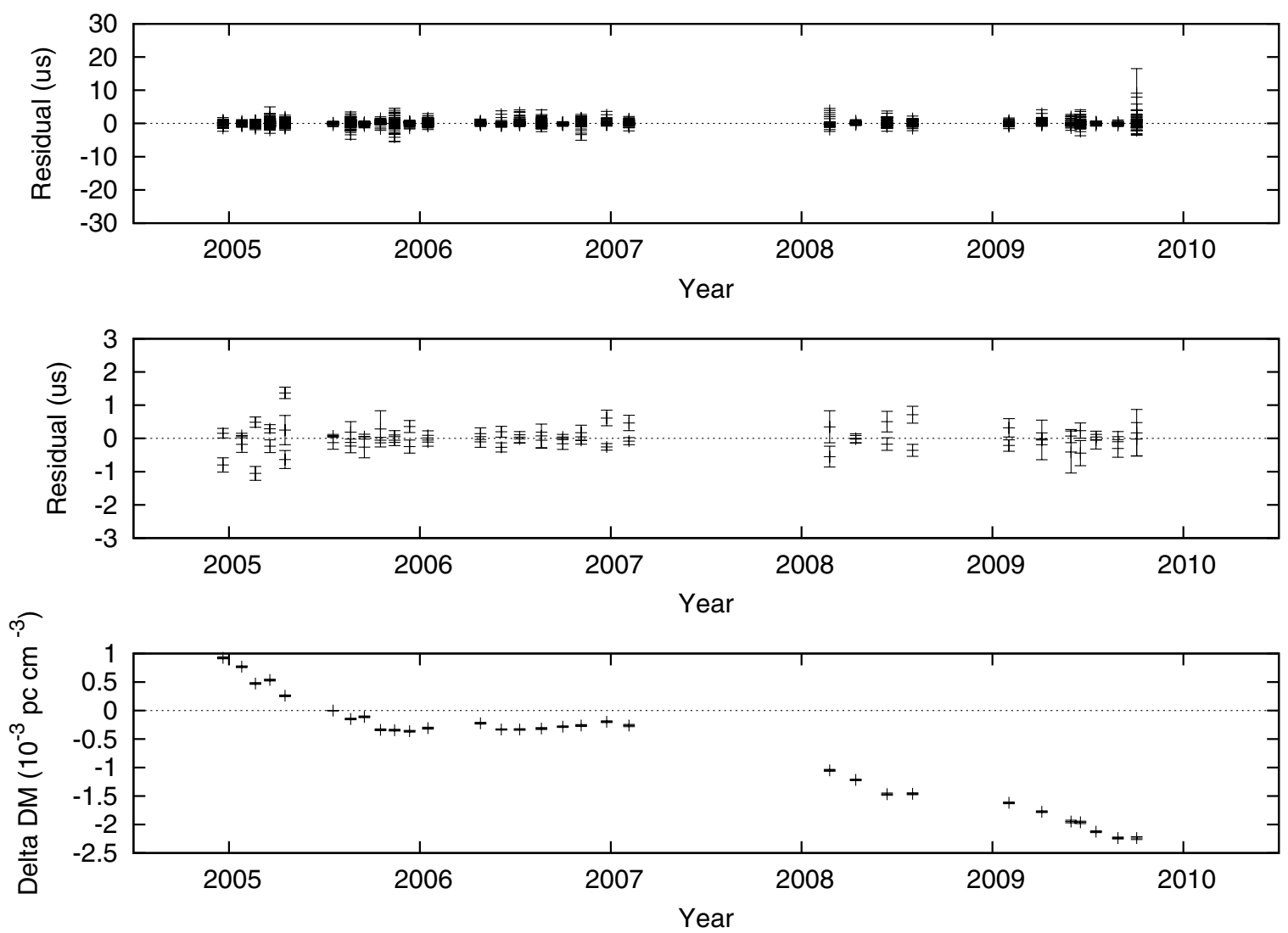

Figure 19. Timing summary for PSR J2317+1439, see Figure 3 for details. 
of cubic and higher order (Kaspi et al. 1994; Jenet et al. 2006). These are approximately orthogonal to the rest of the timing fit, and capture most of the low-frequency GW power. Recent analyses by van Haasteren et al. $(2009,2011)$ use a Bayesian framework to limit or detect GW signals in the data, marginalizing over the timing model parameters. Yardley et al. (2011) use an extensive series of simulations to characterize the effect of timing parameter fitting on the GW signal in the frequency domain.

In this work, we perform the GW analysis in a separate step from the timing fit, while using the properties of the timing fit to both determine how much GW power is absorbed by it, and how to best detect or limit that which remains. This is built around a computation of the statistics (covariance matrix) of the post-fit residuals. This analysis draws heavily on that presented by Demorest (2007), which can be referred to for additional discussion. Further detailed discussion and application of this method will be presented by J. Ellis et al. (2012, in preparation). It is also interesting to note that a very similar calculation appears in the Bayesian method presented by van Haasteren et al. (2009), as a means of marginalizing over the timing fit "nuisance" parameters.

To begin we note that the fit that determines the pulsar parameters is a weighted least-squares minimization, which in matrix terms is the solution of the normal equations (see, for example, Press et al. 1992):

$$
\mathbf{A}^{T} \mathbf{W}^{2} \mathbf{A} \mathbf{a}=\mathbf{A}^{T} \mathbf{W}^{2} \mathbf{y} .
$$

Here, $\mathbf{y}$ is the vector of input data (sometimes called "prefit residuals"), $\mathbf{A}$ is the fit design matrix whose columns are the fit basis functions, $\mathbf{a}$ is the vector of fit parameter values, and $\mathbf{W}$ is the weighting matrix. In a standard weighted leastsquares fit such as is used in this paper, $\mathbf{W}$ is a diagonal matrix whose entries are calculated from the TOA uncertainties, $W_{i i}=\sigma_{i}^{-1}$. However, we write Equation (4) in this general form to emphasize that the following methods are equally valid for a generalized least-squares fit in which $\mathbf{W}$ can have non-zero off-diagonal elements. The generalized approach has recently been proposed for pulsar timing analyses as a way of handling correlated noise in the timing data (Coles et al. 2011). In the generalized case, $\mathbf{W}^{2}$ is the inverse of a non-diagonal noise covariance matrix.

The post-fit residuals $\mathbf{r}=\mathbf{y}-\mathbf{A a}=\mathbf{R y}$ are therefore a linear function of the input data, and can be determined by applying the residual projection operator $\mathbf{R}$ :

$$
\mathbf{R}=\mathbf{I}-\mathbf{A}\left(\mathbf{A}^{T} \mathbf{W}^{2} \mathbf{A}\right)^{-1} \mathbf{A}^{T} \mathbf{W}^{2} .
$$

A key feature of writing the analysis in this form is that although $\mathbf{R}$ does depend of the weighting matrix $\mathbf{W}$, it does not depend on the data values $\mathbf{y}$. Therefore, for a given pattern of weights (or uncertainties), the action of the fit can be studied independent of a specific data realization. This feature can be used to eliminate dependence on simulation in interpreting results and determining GW limits, and can provide additional insight into the properties of the fit procedure. In particular, given a known or assumed input data covariance matrix $\mathbf{C}_{\mathbf{y}}$, the resulting residual covariance matrix $\mathbf{C}_{\mathbf{r}}$ can be easily calculated:

$$
\mathbf{C}_{\mathbf{r}}=E\left\{\mathbf{r r}^{T}\right\}=\mathbf{R} E\left\{\mathbf{y} \mathbf{y}^{T}\right\} \mathbf{R}^{T}=\mathbf{R} \mathbf{C}_{\mathbf{y}} \mathbf{R}^{T} .
$$

The expected cross-covariance between the timing residuals of a pair of pulsars ( $a$ and $b$ ) can be calculated similarly:

$$
\mathbf{C}_{\mathbf{r}_{\mathbf{a}}, \mathbf{r}_{\mathbf{b}}}=E\left\{\mathbf{r}_{\mathbf{a}} \mathbf{r}_{\mathbf{b}}^{T}\right\}=\mathbf{R}_{\mathbf{a}} \mathbf{C}_{\mathbf{y}_{\mathbf{a}}, \mathbf{y}_{\mathbf{b}}} \mathbf{R}_{\mathbf{b}}^{T} \text {. }
$$

The procedure presented here for determining post-fit covariance and cross-covariance matrices accounts for GW signal power removed by all terms included in the timing model fit. In the timing analysis presented in this work, this includes the time-variable DM terms and interfrequency offsets, as well as the usual spin, astrometric and binary parameters. In general, increasing the number of timing fit parameters will reduce the amplitude of a GW signal in the residuals and hence reduce the overall GW sensitivity of the experiment.

It is worth noting that the ensemble of post-fit residuals is in general non-stationary. That is, the elements of the covariance matrix, $C_{r, i j}$, are not simply a function of the time lag $\tau_{i j}=$ $t_{j}-t_{i}$, even if the pre-fit stochastic process represented by $\mathbf{C}_{\mathbf{y}}$ does have this property. Therefore, the action of the timing fit cannot be fully represented as a filter or frequency domain transfer function, as has sometimes been proposed in the past (e.g., Blandford et al. 1984; Jenet et al. 2005). This feature of the statistics of the residuals, along with the irregular sampling, varying data span and quality, and presence of steep-spectrum red noise processes, present significant obstacles to a frequency domain analysis of real-world pulsar timing data. This motivates our decision to perform the $\mathrm{GW}$ analysis in the time domain.

\subsection{Single-pulsar Analysis}

We can use the methods discussed in the previous section, along with timing data from a single pulsar, to compute upper limits on the strength of the GWB. As previously discussed, there is no definitive way to distinguish GW-induced timing fluctuations observed in a single pulsar from those due to intrinsic pulsar spin irregularity or other non-GW effects. However, in the low-amplitude GW regime, focusing on the single best pulsar in this manner can provide some of the most constraining upper limits.

Given a GW spectrum of assumed power-law shape but unknown amplitude, we compute the pre-fit GW covariance matrix $\mathbf{C}_{\mathbf{y}}^{\mathbf{G W}}$ up to an overall scaling by $A_{1}^{2}$ (see Equation (3) and van Haasteren et al. 2009). This is then converted to the residual covariance matrix $\mathbf{C}_{\mathbf{r}}^{\mathbf{G W}}$ using Equation (6). Diagonalizing the weighted covariance matrix $\mathbf{W C}_{\mathbf{r}}^{\mathbf{G W}} \mathbf{W}$ provides an orthonormal basis of eigenvectors that can be used to decompose the timing residuals. This basis represents the GW signal using the smallest possible number of components, ${ }^{28}$ and is completely orthogonal to the timing fit. In other words, this basis optimally captures the portion of the GW signal in the data that is not absorbed by the timing model fit. Transforming the residuals into this basis produces a set of projection coefficients $c_{i}$. The associated eigenvalues $\left(\lambda_{i}\right)$ give the relative level of $\mathrm{GW}$ signal power in each component, and by assuming a nominal value of $A_{1}$, can be scaled to produce expected mean-square timing residual values. Figure 20 shows the measured and expected component amplitudes for the two best pulsars in our set, PSRs J1713+0747 and $\mathrm{J} 1909-3744$, assuming $\alpha=-2 / 3$ and $A_{1}=10^{-15}$. In these plots and the following discussion, the residual coefficients and eigenvalues have been normalized to represent the rms residual due to each component. The quadrature sum of all coefficients for a given pulsar $\left(\sum c_{i}^{2}\right)^{1 / 2}$ reproduces its full rms residual as presented in Table 2 .

It is immediately apparent that the data shown in a plot such as Figure 20 can be used to place a limit on the strength of a

\footnotetext{
28 This is very similar to principal components analysis (PCA). The only difference is that PCA is typically based on an empirical data covariance matrix rather than an assumed covariance matrix as we have used here.
} 

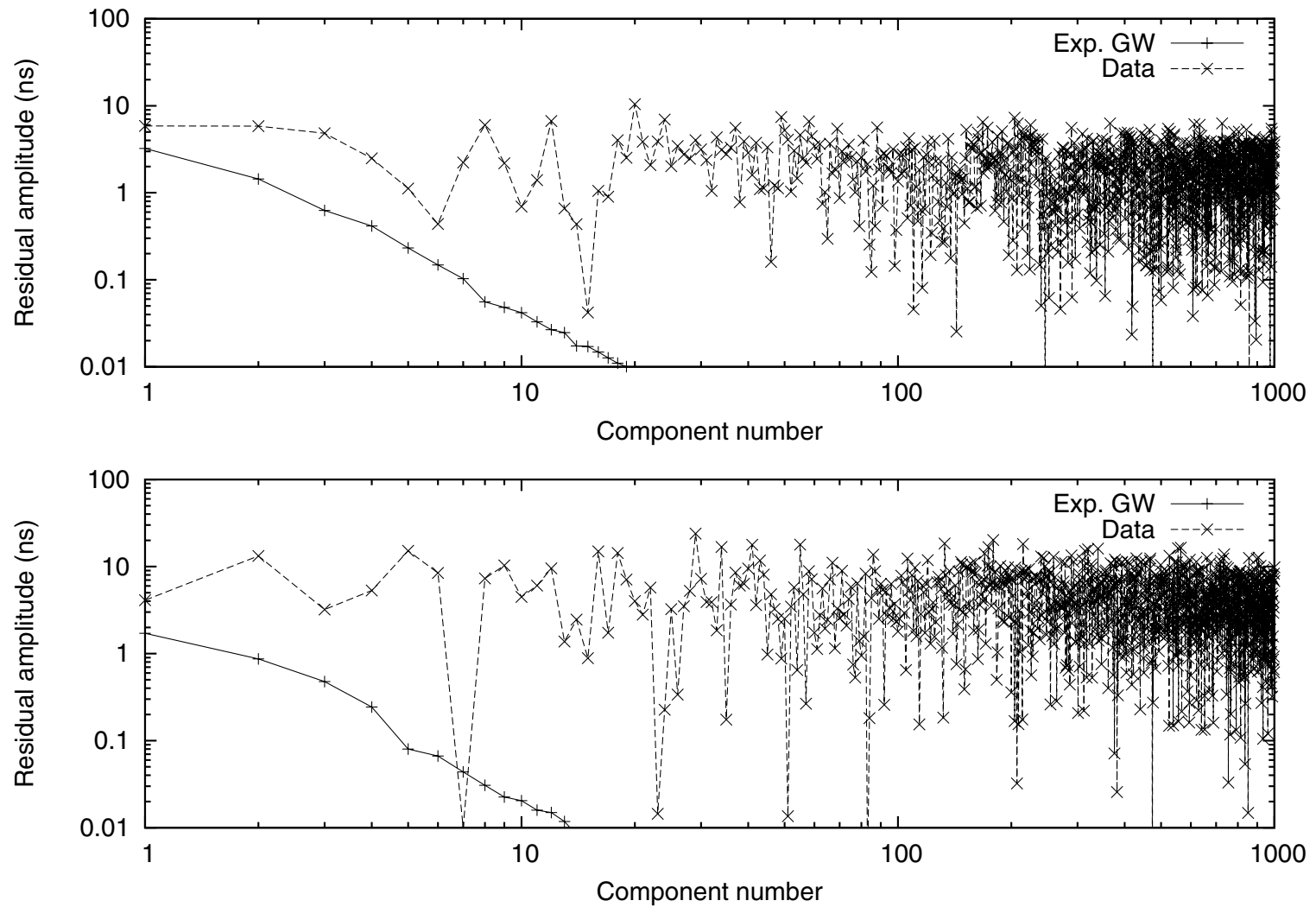

Figure 20. Eigenvalue spectra for J1713+0747 (top) and J1909-3744 (bottom). In each plot, the line labeled "Data" shows the absolute value of the residual coefficients $c_{i}$ when transformed into the optimal basis for an $\alpha=-2 / 3$ power-law GW spectrum. The "Exp. GW" line shows the expected amplitude (square root of the eigenvalues, $\lambda_{i}^{1 / 2}$ ) for an $A_{1}=10^{-15} \mathrm{GW}$ signal level. The fact that the data lines are flat reflects the fact that the residuals are dominated by white noise.

power-law GWB. Roughly speaking, any GW signal present in the data must lie near or below the observed data values. For measured component values $c_{i}$ and eigenvalues $\lambda_{i}$, we combine several components into a single limit by forming the likelihood function for the parameters $A_{1}$ and $\sigma$, the level of white noise present in the data:

$$
\log L\left(A_{1}, \sigma\right)=-\frac{1}{2} \sum_{i}\left\{\log 2 \pi\left(A_{1}^{2} \lambda_{i}+\sigma^{2}\right)+\frac{c_{i}^{2}}{A_{1}^{2} \lambda_{i}+\sigma^{2}}\right\} .
$$

This formulation assumes both the GW signal and the white noise follow Gaussian statistics; in this case each component value $c_{i}$ is drawn from a normal distribution with zero mean and variance equal to $A_{1}^{2} \lambda_{i}+\sigma^{2}$. The different components are by construction independent due to the choice of basis. The product of all the component distributions for a given pulsar results in the likelihood function of Equation (8). Using uniform/positive priors on $\sigma$ and $A_{1}$, and marginalizing over $\sigma$, we convert the likelihood function above into a posterior probability distribution for $A_{1}$, shown in Figure 21. For the data from $\mathrm{J} 1713+0747,95 \%$ of the distribution falls under $A_{1}<1.1 \times 10^{-14}$ for an $\alpha=-2 / 3$ spectrum. This is the most constraining single pulsar in our set. Similar upper limits computed for each of the pulsars at a variety of astrophysically relevant $\alpha$ are listed in Table 3.

While we have framed the discussion so far in terms of a GWB signal, this analysis method is more fundamentally a way to quantify timing perturbations of a known/assumed spectral shape that may exist in the data, regardless of their cause. In this context we can look at these single-pulsar results as a test for "red" power-law timing noise in the data set. In addition to the $95 \%$ upper limit on $A_{1}$ already described, two other statistics are useful: The maximum likelihood estimate $\hat{A_{1}}$ and the ratio $R=L\left(0, \hat{\sigma_{0}}\right) / L\left(\hat{A_{1}}, \hat{\sigma}\right)$ between the maximum likelihood values obtained by either fixing $A_{1}=0$ or allowing it to vary. $\hat{A_{1}}$ characterizes the strength of the red noise, and $R$ characterizes its significance in the data. Values of $R$ near 1 indicate consistency with a white-noise-only model, while very small values of $R$ indicate significant red noise is detected. These three values are listed for all pulsars for a selection of various expected GW spectral indices in Table 3. We see that two of the pulsars-J1643-1224 and J1910+1256-show very significant red noise $(R<0.01)$, and two others- $\mathrm{J} 1640+2224$ and B1953+29-show mildly significant red noise $(0.01 \lesssim R<$ 0.1 ). The remaining 13 sources are consistent with white noise only. Additional detailed analyses of the noise in these data are ongoing, and include both a Bayesian analysis covering a wider range of power-law spectral index (J. Ellis et al. 2012, in preparation), and an application of the Coles et al. (2011) methodology to our data set (D. Perrodin et al. 2012, in preparation).

We will not attempt to definitively determine the cause of the red noise in the four sources noted here. However, we speculate that at least for three of them a likely source is the interstellar medium, rather than processes intrinsic to the pulsars. As explained above, J1910+1256 and B1953+29 do not include $\operatorname{DM}(t)$ fits so their timing will be affected at some level by unmodeled DM variation. J1643-1224 has the second highest DM of any pulsar in our set (after B1953+29), and has a high predicted level of interstellar scatter-broadening, suggesting that multipath ISM effects may be an issue here. 


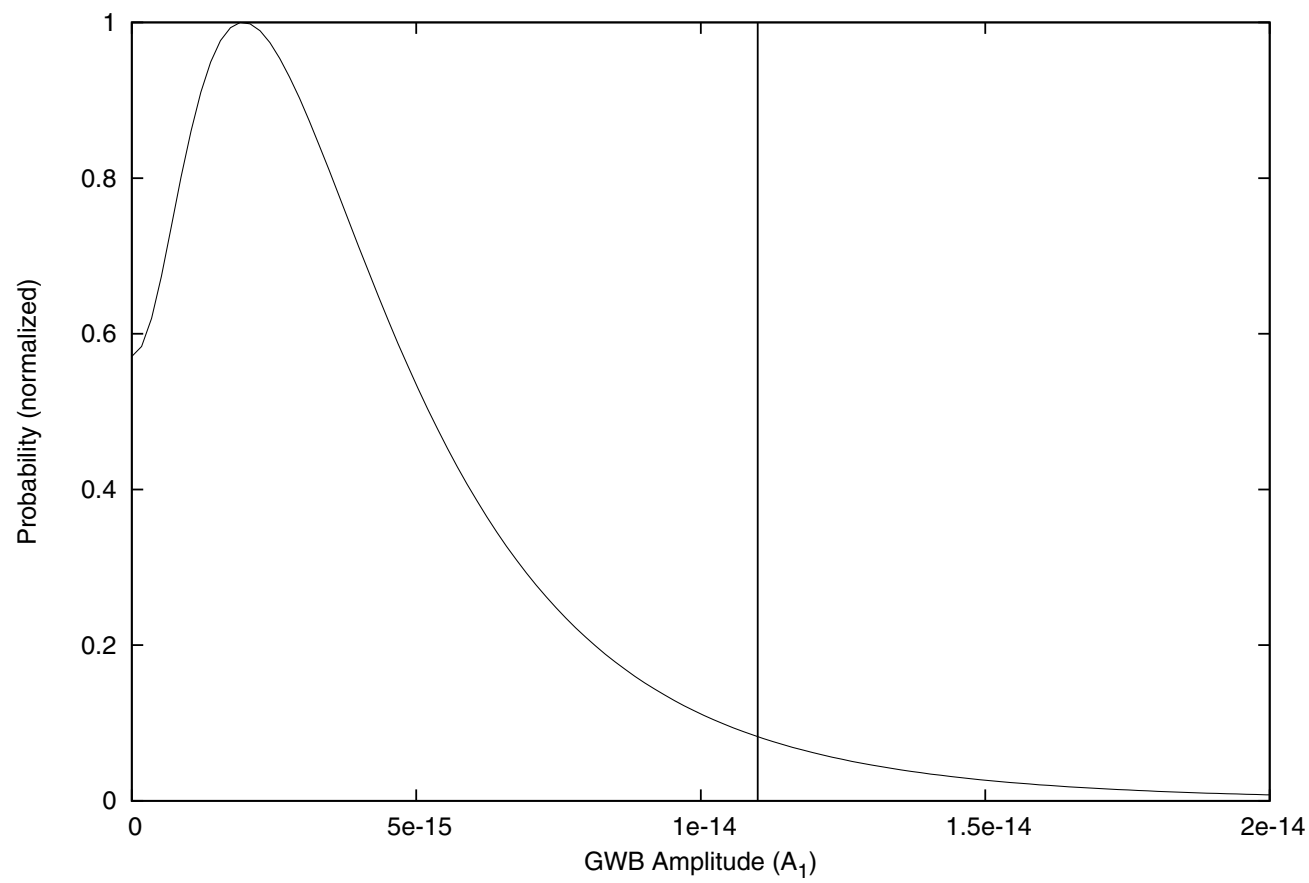

Figure 21. Probability distribution for the GWB amplitude $A_{1}$ based on $\mathrm{J} 1713+0747$ timing, and assuming $\alpha=-2 / 3$. 95\% of the distribution is contained in $A_{1}<1.1 \times 10^{-14}$ (vertical line), the maximum likelihood value $\hat{A_{1}}=1.9 \times 10^{-15}$, and the likelihood ratio between this point and $A_{1}=0$ is $R=0.6$. In this case, a non-zero value of $A_{1}$ provides the best fit to the data, but without much statistical significance over a white-noise-only model. These statistics for all sources, considering several different values of $\alpha$, are presented in Table 3 .

Table 3

Results from Single-pulsar GW Analyses

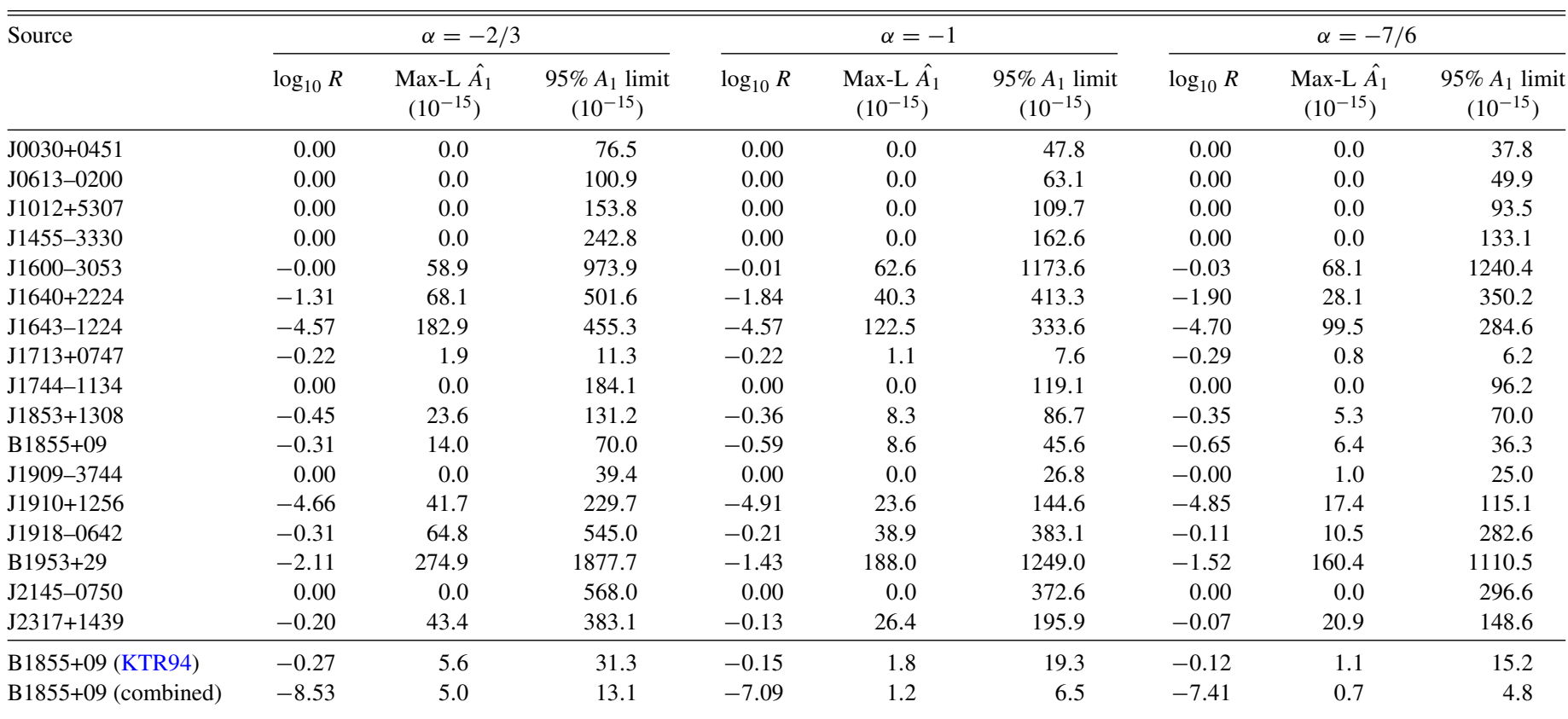

\subsection{Verification}

The methods discussed in the previous section do not rely at all on simulated data to produce a GW upper limit or red noise estimate. However, it is still a valuable exercise to analyze a simulated GW signal using this approach, as a test of its validity. To do so, we added simulated GW to our timing data for J1713+0747, using the TEMPO2 GWBKGRD plugin (Hobbs et al. 2009). GW were added using $\alpha=-2 / 3$ and $A_{1}=10^{-14}$. These data were then analyzed using the eigenvalue procedure described above. Figure 22 shows the rms component values $\left(c_{i}\right)$ from $1000 \mathrm{GW}$ realizations, along with the square root of the associated eigenvalues $\left(\lambda_{i}^{1 / 2}\right)$ scaled to the known $\mathrm{GW}$ signal level. The excellent agreement between these two lines shows that our algorithm is correctly predicting the amount of GW power present in the timing residuals. Figure 23 presents results of computing maximum likelihood and $95 \%$ upper limit amplitude values from the simulated data. These statistics agree well with the known amount of GW signal in the simulated data (gray region in the plots). 


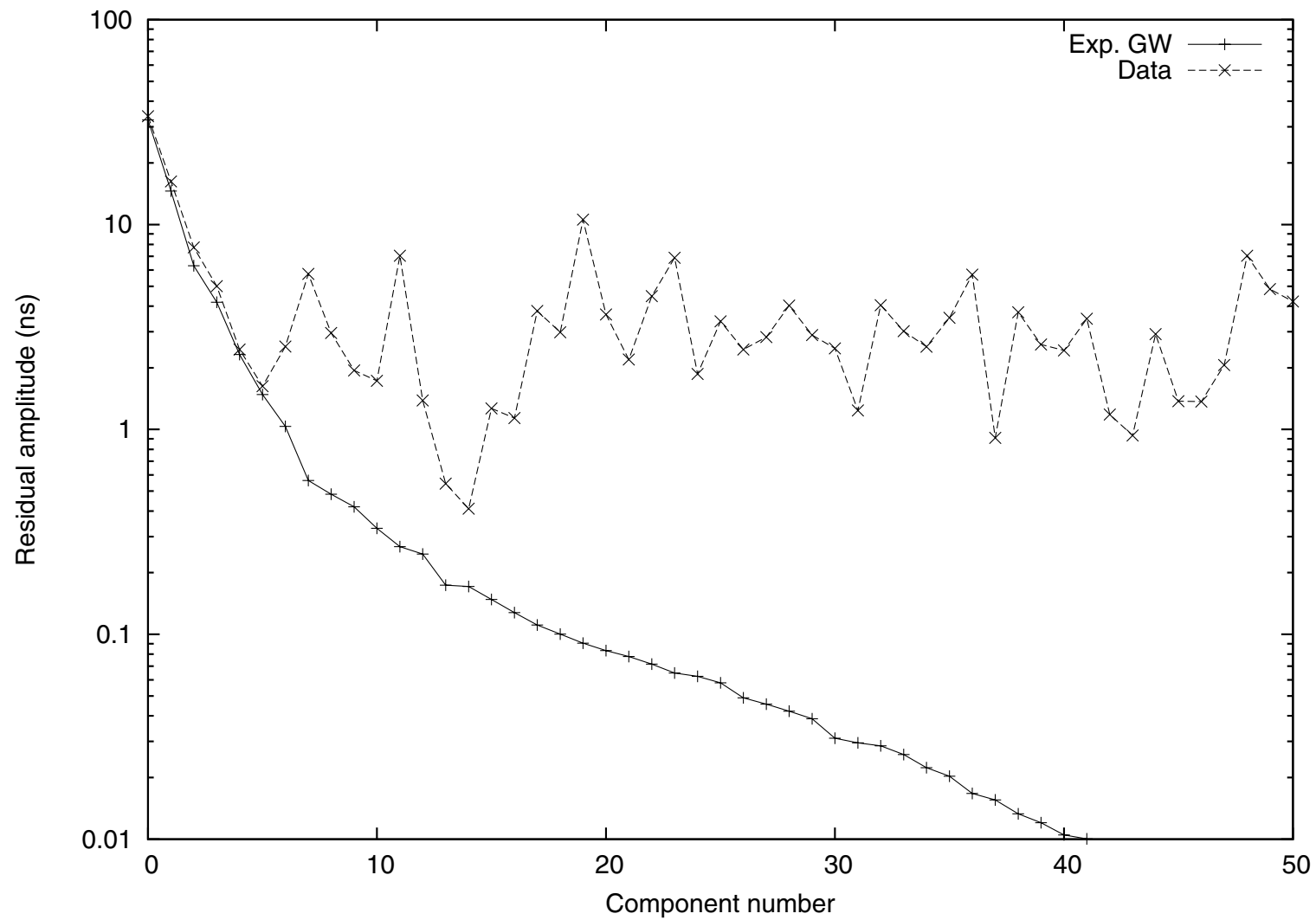

Figure 22. Eigenvalue spectra for J1713+0747 with simulated GW injected at an amplitude of $A_{1}=10^{-14}$. As in Figure 20, the "Exp. GW" line shows the expected GW amplitude determined from the eigenvalue analysis, scaled to the known signal level. The "Data" line shows the root-mean-square residual in each component, averaged over 1000 realizations of the simulation. The correct GW signal level is observed in the data, until the level drops below the white noise near component 7.
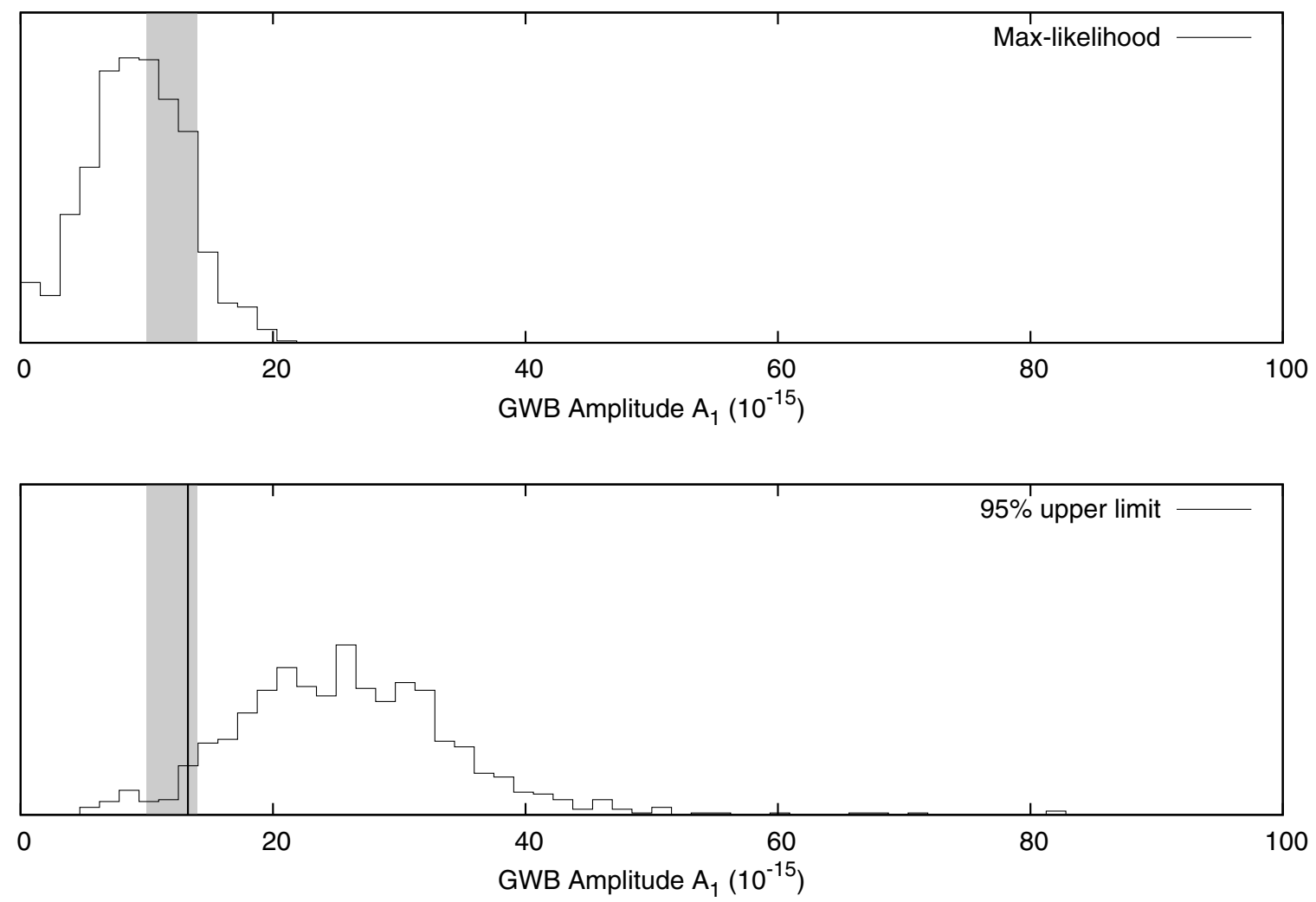

Figure 23. Histograms of maximum likelihood GW amplitude (upper panel), and 95\% upper limit (lower panel) computed from 1000 realizations of a simulated $A_{1}=10^{-14}, \alpha=-2 / 3 \mathrm{GWB}$ added to J1713+0747 timing data. The shaded range represents the true GW signal level, including both the simulated signal and any existing GW signal in the data, up to the limit presented in Section 4.2. The vertical line in the lower panel shows the 5\% quantile of the histogram values. 
As an additional test of this method, we analyzed the publicly available seven-year B1855+09 timing data set published by Kaspi et al. (1994, hereafter KTR94), from observations taken during 1986-1992. This data set has been used for several previous GW analyses, so provides a useful benchmark for testing different analysis methods. For this comparison we focus on the GW spectrum originally considered by KTR94, with $\alpha=-1$. From the KTR94 data set alone we find no significant evidence for red timing noise (see results in Table 3), and using our method it sets a $95 \%$ upper limit of $A_{1}<1.9 \times 10^{-14}$. This is somewhat more conservative than the published limits of $A_{1}<1.3 \times 10^{-14}$ (KTR94) and $A_{1}<1.4 \times 10^{-14}$ (Jenet et al. 2006) obtained from the same data. ${ }^{29}$ This difference may be due to the varying strategies used by these three analyses to account for effects of the timing model fit and the possibility of existing red noise in the data.

Combining the KTR94 data with our newer observations of this pulsar into a single analysis produces a limit $A_{1}<$ $6.5 \times 10^{-15}$, comparable to what we get from our $\mathrm{J} 1713+0747$ data alone. However, very significant red noise is detected in the combined B1855+09 data set (Table 3). A fundamental feature of red noise processes is that their amplitude grows with increasing data span. Therefore, this detection in the combined data set is not inconsistent with the lack of such a detection in either of the two data subsets on their own. Another contributing factor may be unmodeled variation in DM. The KTR94 data were obtained at a single frequency and as such cannot be corrected for DM variation. In our newer data, a significant $\operatorname{DM}(t)$ trend is clearly visible for this pulsar (Figure 13).

\subsection{Cross-correlation Analysis}

To detect a GW signal present in the timing data, we must consider correlations among the timing residuals of different pulsars, as previously discussed. This is complicated by the fact that residuals from each pulsar are produced by separate timing model fits. The fits potentially incorporate a wide variety of effects (e.g., binary parameters) that vary from pulsar to pulsar. Furthermore the span and cadence of observations is not necessarily identical between all the sources, and each may be affected by a different level of red timing noise from either $\mathrm{GW}$ or other sources. Correctly measuring the correlation between two such residual timeseries is therefore more complicated than simply multiplying and averaging the two signals, as would be done in a standard correlation analysis.

Referring to the discussion in Section 4.1 and in particular Equation (7), given the actual timing model fits used for any two pulsars and an expected gravitational wave spectrum, we determine the expected cross-correlation for the $\mathrm{GW}$ signal between all pairs of residuals of each pulsar pair $\left(\mathbf{C}_{\mathbf{r}_{\mathbf{a}}, \mathbf{r}_{\mathbf{b}}}^{\mathbf{G W}}\right)$. We use the elements of this matrix, along with the estimated noise parameters from Section 4.2 as weights for a cross-correlation sum to estimate the GW signal power correlated between pulsars $a$ and $b$ :

$$
\rho_{a b}=\frac{\sum_{\mathrm{ijkl}} r_{i}^{(a)}\left(C^{\operatorname{tot}(a)}\right)_{i j}^{-1} C_{j k}^{\mathrm{GW}(a, b)}\left(C^{\mathrm{tot}(b)}\right)_{k l}^{-1} r_{l}^{(b)}}{\sum_{\mathrm{ijkl}}\left(C^{\operatorname{tot}(a)}\right)_{i j}^{-1} C_{j k}^{\mathrm{GW}(a, b)}\left(C^{\mathrm{tot}(b)}\right)_{k l}^{-1} C_{i l}^{\mathrm{GW}(a, b)}} .
$$

The components of this expression are as follows:

1. The sum indices $i$ and $j$ run from 1 to $N_{a}$, the number of TOAs for pulsar $a$. Similarly, $k$ and $l$ run from 1 to $N_{b}$.

\footnotetext{
${ }^{29}$ Limits originally published in units of $\Omega_{g w} h^{2}$ were converted to equivalent
} $A_{1}$ using Equation (3) of Jenet et al. (2006).
2. $r_{i}^{(a)}$ and $r_{l}^{(b)}$ are simply the post-fit timing residuals for pulsars $a$ and $b$, respectively.

3. $C_{i j}^{\text {tot }(a)}$ and $C_{k l}^{\text {tot }(b)}$ are the total post-fit covariance matrices for each pulsar. These are determined using the maximum likelihood red noise parameters found in the earlier singlepulsar analysis, combined with the TOA measurement uncertainties, and modified by the timing model fit (see Equation (6)):

$$
\mathbf{C}_{\mathbf{r}}^{\text {tot }}=\mathbf{R}\left({\hat{A_{1}}}^{2} \mathbf{C}_{\mathbf{y}}^{\mathbf{G W}}+\mathbf{W}^{-2}\right) \mathbf{R}^{T}
$$

4. $C_{j k}^{\mathrm{GW}(a, b)}$ are the elements of $\mathbf{C}_{\mathbf{r}_{\mathbf{a}}, \mathbf{r}_{\mathbf{b}}}^{\mathbf{G W}}$, the expected post-fit cross-covariance matrix for the GW signal in pulsars $a$ and $b$, computed using Equation (7) and the assumed power-law spectral shape.

The presence of the inverse covariance matrices in Equation (9) effectively serves to "whiten" the timing residuals before computing the cross-correlation sum. This reduces the weight of data from pulsars that have red noise components, potentially including any "self-noise" contribution from the stochastic GWB itself. This formulation is conceptually very similar to the frequency-domain optimal statistic presented by Anholm et al. (2009).

The post-fit covariance matrices $\mathbf{C}_{\mathbf{r}}^{\text {tot }}$ are singular, due to the degrees of freedom removed by the timing fit, or equivalently by the application of the $\mathbf{R}$ operator. Therefore, the matrix inverse technically does not exist. In this situation it is appropriate to use a singular value decomposition-based pseudoinverse in its place. This is equivalent to performing the correlation in the subspace of signals that are orthogonal to (i.e., not absorbed by) the timing fit. The uncertainty on $\rho_{a b}$ is given by

$$
\sigma_{\rho_{a b}}=\left(\sum_{\mathrm{ijkl}}\left(C^{\mathrm{tot}(a)}\right)_{i j}^{-1} C_{j k}^{\mathrm{GW}(a, b)}\left(C^{\mathrm{tot}(b)}\right)_{k l}^{-1} C_{i l}^{\mathrm{GW}(a, b)}\right)^{-1 / 2} .
$$

The resulting $\rho_{a b}$ for all 136 pulsar pairs, with $\alpha=-2 / 3$, is shown in Figure 24. We search for the presence of the GWB in these data by fitting them to an amplitude (proportional to $A_{1}^{2}$ ) times the Hellings-Downs angular function $\zeta\left(\theta_{a b}\right)$. The best fit, also shown in Figure 24 , results in $A_{1}^{2}=(-10 \pm 26) \times 10^{-30}$ for $\alpha=-2 / 3$. This is consistent with no detectable GWB in the data, and also can be interpreted as a $2 \sigma$ upper limit of $A_{1}<7.2 \times 10^{-15}$, an improvement over the single-pulsar limits presented in Section 4.2. The reduced $-\chi^{2}$ of this fit is 0.95 , providing additional confidence that the uncertainty estimate of Equation (11) is correct.

A table of the top 15 correlation measurements, sorted by increasing uncertainty, is presented in Table 4. It is clear that the measurement is dominated by pairs involving one or both of the two best-timing pulsars in the set, J1713+0747 and J1909-3744. In total, 8 separate pulsars contribute to these 15 lowest-uncertainty points. The limit being set primarily by a small number of pulsars is due to the fact that our current data set is in a white noise dominated, low GW amplitude regime. As the length and quality of such data sets increase, they are expected to become increasingly dominated by red noise components, either from the GWB itself or from intrinsic pulsar timing noise. In the red noise dominated and/or strong GW regime, a large number of pulsars (likely $\sim 20-50$ ) is required to improve the significance of a GW detection (Jenet et al. 2005; Cordes $\&$ Shannon 2012). This provides motivation for continuing to 


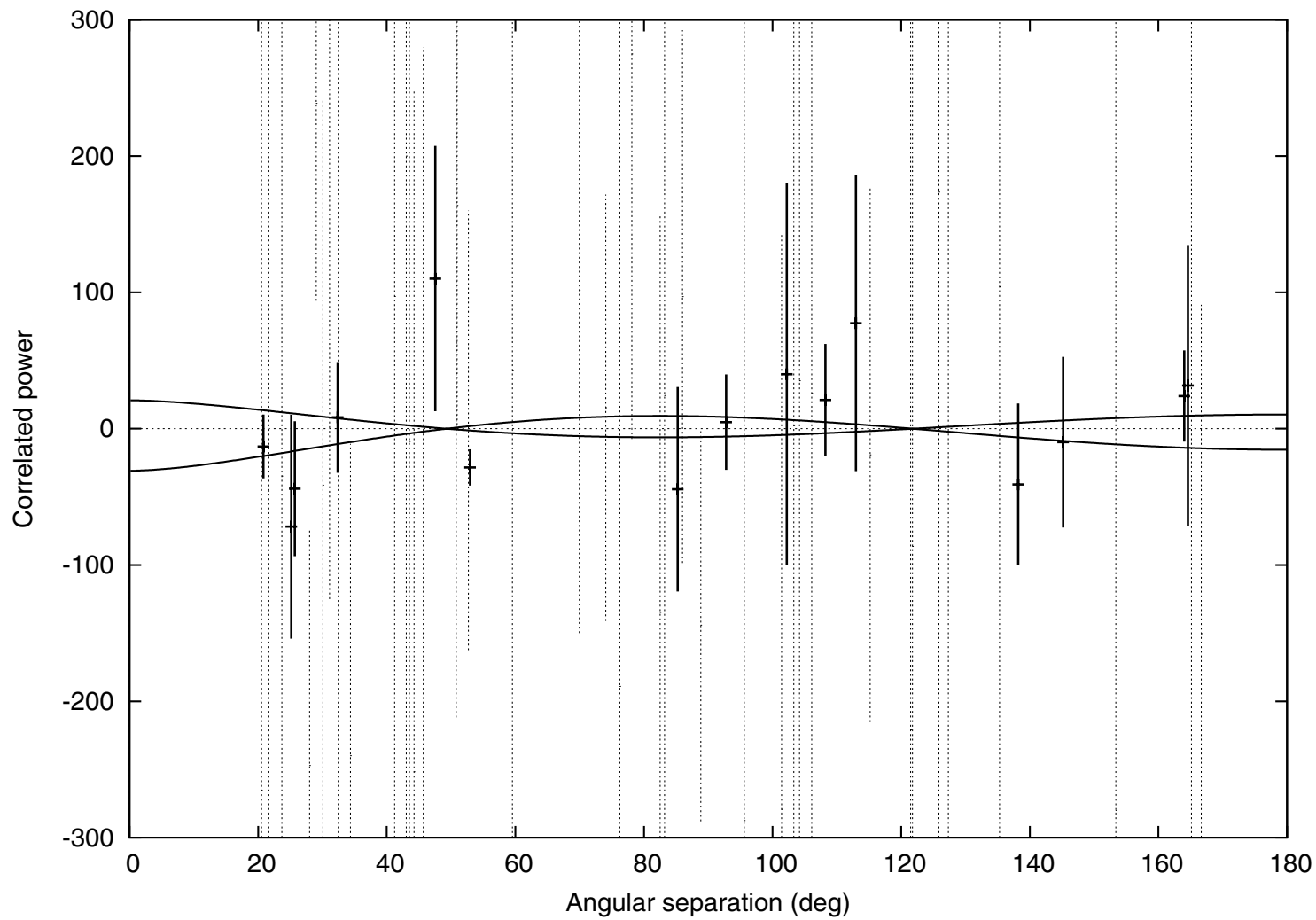

Figure 24. Measured cross-correlated power $\rho_{a b}$ as a function of separation angle $\theta_{a b}$ for pairs of pulsars in our set, with error bars showing $1 \sigma$ uncertainty. Power is normalized relative to an $A_{1}^{2}=10^{-30}, \alpha=-2 / 3$ GWB. The lines show the $\pm 2 \sigma$ fit to the amplitude of the Hellings-Downs function $\zeta(\theta)$. All 136 cross-correlation points were used for the fit, however for clarity the 15 lowest-uncertainty values are denoted with solid/bold symbols.

Table 4

Cross-correlated Power Measurements and GW Results

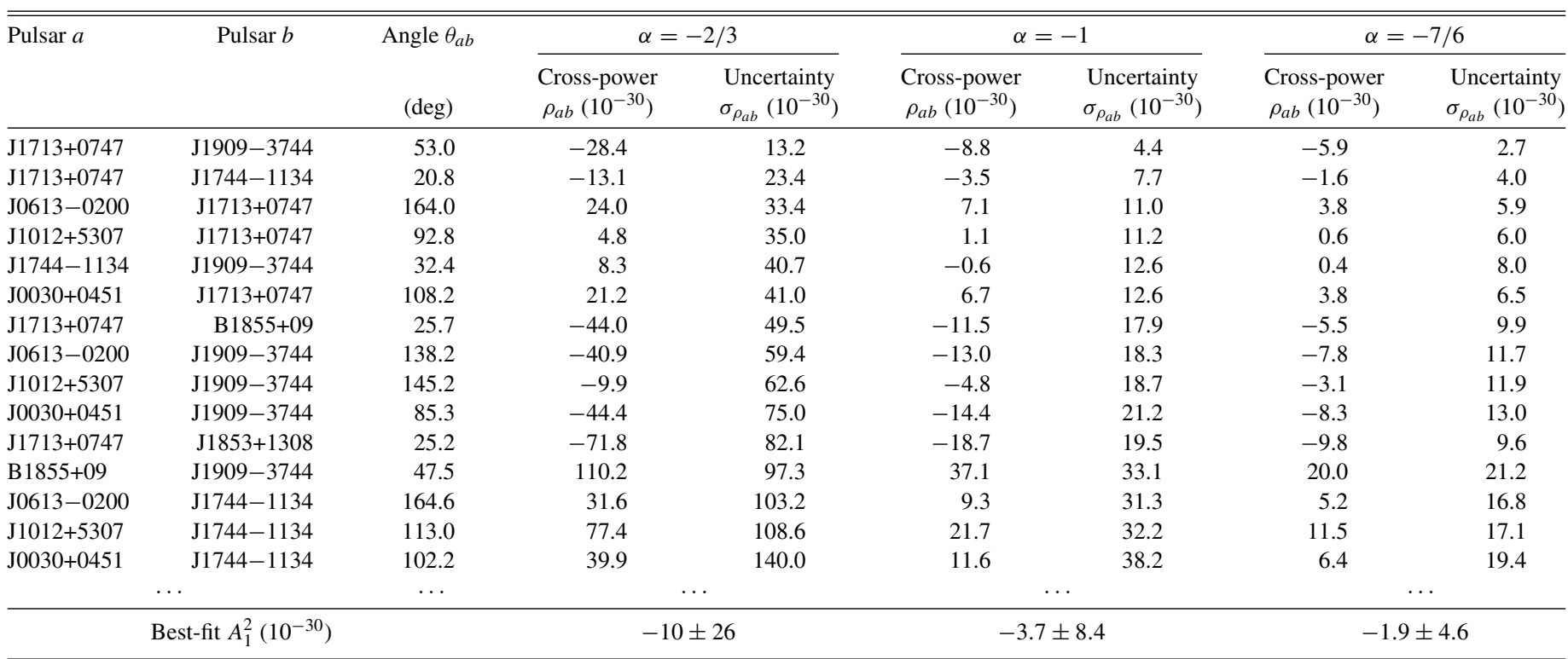

observe many MSPs, even if upper limits are currently only dominated by a small subset of the pulsars.

Table 4 also lists the best-fit $A_{1}^{2}$ and its uncertainty for three different spectral indices. The resulting $2 \sigma$ upper limits are $A_{1}=7.2 \times 10^{-15}, 4.1 \times 10^{-15}$, and $3.0 \times 10^{-15}$ for $\alpha=-2 / 3$, -1 , and $-7 / 6$, respectively. The change in value of $A_{1}$ with $\alpha$ is primarily due to the fact that although we are using 1 year $^{-1}$ as the reference GW frequency, the measurement is most sensitive to frequencies near $T^{-1}$, where $T \sim 5$ years is the effective length of the multi-pulsar data set. Therefore, we expect the various limits on $A_{1}$ to scale with $\alpha$ as follows:

$$
A_{1}(\alpha)=A_{T}\left(\frac{T}{1 \text { year }}\right)^{\alpha} \text {. }
$$

Using our three measured $A_{1}$ limits to empirically determine $A_{T}$ and $T$ gives $A_{T}=2.26 \times 10^{-14}$ and $T=5.54$ years (see Figure 25). These values can be used together with Equation (12) 


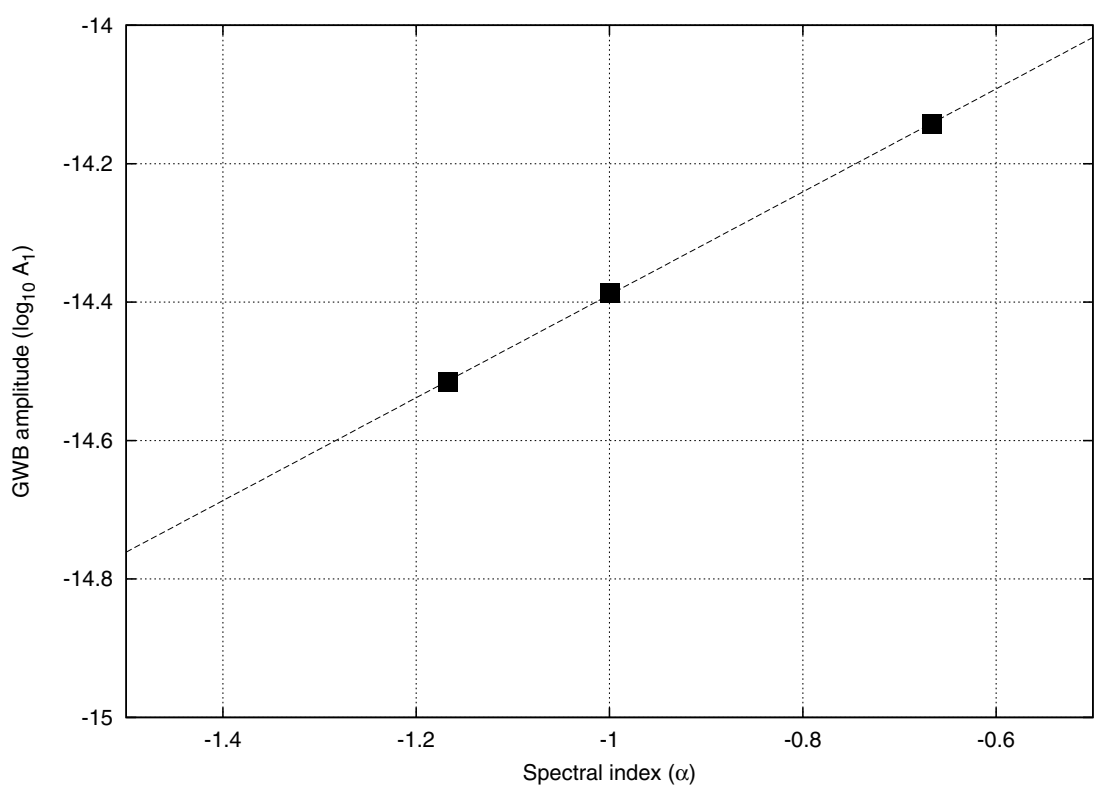

Figure 25. Measured $2 \sigma$ upper limits on $A_{1}$ as a function of $\mathrm{GW}$ spectral index $\alpha$ (squares). The values are consistent with a simple scaling based on $A_{T}=2.26 \times 10^{-14}$ at $T=5.54$ years and Equation (12) (line). This relationship can be used to convert these results to equivalent limits for other values of $\alpha$.

to convert our measurement into an approximate limit on $A_{1}$ for values of $\alpha$ that we have not considered here.

\section{DISCUSSION}

\subsection{Supermassive Black Holes}

As previously mentioned, the strongest expected source of $\mathrm{GW}$ in the nanohertz band is the ensemble background from SMBHs, expected to have a spectral index of $\alpha=-2 / 3$. It is on these which we will focus our discussion here. The GWB predicted from the SMBH binary population is dependent on a number of observationally ill-constrained values: e.g., the ubiquity of SMBHs in galaxy centers, the prevalence of merging, as well as the timescale and dominant mode (growth via accretion or merger with other black holes) of black hole growth. We thus rely on various parameterizations of black hole demographics through which to interpret our background limit.

Importantly, we first endeavor to explicitly rederive the standard parameterization of the predicted characteristic strain spectrum, $h_{c}(f)$, from the cosmological population of binary SMBHs. The literature demonstrates inconsistencies for this parameterization; in particular, we wish to point out that despite performing an apparently equivalent derivation, Equation (32) of Jaffe \& Backer (2003) and Equation (8) of Jenet et al. (2006) differ. In addition, the limits placed by Jenet et al. (2006) on the parameter $I$, defined below, are inconsistent with their reported limit on the characteristic strain.

We begin with the strain amplitude of an SMBH binary system (Peters \& Mathews 1963):

$$
h_{s}=4 \sqrt{\frac{2}{5}} \frac{\left(G M_{c}\right)^{5 / 3}}{c^{4} D} f^{2 / 3}(1+z)^{2 / 3} \pi^{2 / 3},
$$

where $f$ is the GW frequency in the observer's rest frame, $D$ is the distance to the SMBH binary, $z$ is the system's cosmological redshift, and $M_{c}=\left[m_{1} m_{2}\left(m_{1}+m_{2}\right)^{-1 / 3}\right]^{3 / 5}$ is the binary chirp mass. The amount of time $d \tau$ spent per GW spectral bin $d f$ is given by (Peters \& Mathews 1963):

$$
\frac{d \tau}{d f}=\frac{5}{96}\left(\frac{c^{3}}{G M_{c}}\right)^{5 / 3} \pi^{-8 / 3} f^{-11 / 3}(1+z)^{-5 / 3}
$$

In the proper rest frame of any comoving point in space, the number density of SMBHs merging per unit time $\left(t_{p}\right)$ per unit first SMBH mass $\left(m_{1}\right)$ per unit BH mass of its companion $\left(m_{2}\right)$ is written as

$$
Q\left(m_{1}, m_{2}, t_{p}, \boldsymbol{x}\right)=\frac{d N}{d t_{p} d^{3} x d m_{1} d m_{2}},
$$

where $d^{3} x$ represents a unit of comoving volume. We then wish to determine the number of merging events per unit time $t$ observed in the observer rest frame (i.e., $z=0$ ) per unit $z$ per unit $m_{1}, m_{2}$. Taking cosmological considerations into account, this is given by (e.g., Jaffe \& Backer 2003):

$$
v\left(m_{1}, m_{2}, t, z\right)=4 \pi \frac{c}{H_{0}} \frac{D^{2}}{E(z)} \frac{Q}{1+z},
$$

where $H_{0}$ is the Hubble constant, and $E(z)=\sqrt{\Omega_{\Lambda}+\Omega_{m}(1+z)^{3}}$. We set $\Omega_{\Lambda}=0.7$ and $\Omega_{m}=0.3$ to represent a standard flat universe.

Given $h_{s}, d \tau / d f$, and $v\left(m_{1}, m_{2}, t, z\right)$, we can construct the expected strain per unit $f$ induced by all binary black holes in the universe, giving the characteristic strain spectrum:

$$
\begin{aligned}
h_{c}(f)^{2} & =\int f h_{s}^{2} \frac{d \tau}{d f} v\left(m_{1}, m_{2}, z\right) d m_{1} d m_{2} d z \\
& =\frac{4}{3 \pi^{1 / 3} c^{2}} \frac{G^{5 / 3}}{H_{0} f^{4 / 3}} \int Q \frac{M_{c}^{5 / 3}}{(1+z)^{4 / 3} E(z)} d m_{1} d m_{2} d z .
\end{aligned}
$$

We then assume that $Q$ can be written as

$$
Q=\phi\left(m_{1}, m_{2}\right) R\left(t_{p}, \boldsymbol{x}\right),
$$


where $\phi\left(m_{1}, m_{2}\right)$ is the mass function of SMBHs, and $R\left(t_{p}, \boldsymbol{x}\right)$ is the total number of coalescing binary systems per unit time in the binary rest frame. Note that we explicitly assume in Equation (18) that $\phi$ is not redshift-dependent. We then regroup these terms to state:

$$
\begin{aligned}
\left\langle M_{c}^{5 / 3}\right\rangle & =\int \phi\left(m_{1}, m_{2}\right) M_{c}^{5 / 3} d m_{1} d m_{2} \\
I & =\int \frac{R\left(t_{p}, \boldsymbol{x}\right)}{R_{0}} \frac{1}{(1+z)^{4 / 3} E(z)} d z,
\end{aligned}
$$

where $R_{0}$ is $R\left(t_{p}, \boldsymbol{x}\right)$ evaluated at the location of Earth at the current epoch in time. Using the above definitions, we arrive at the final form of our characteristic strain spectrum:

$$
h_{c}(f)^{2}=\frac{4}{3 \pi^{1 / 3} c^{2}} \frac{G^{5 / 3}}{H_{0} f^{4 / 3}}\left\langle M_{c}^{5 / 3}\right\rangle R_{0} I .
$$

We adopt the following values:

$$
\begin{aligned}
c & =2.998 \times 10^{8} \mathrm{~m} \mathrm{~s}^{-1} \\
G & =6.673 \times 10^{-11} \mathrm{~m}^{3} \mathrm{~kg}^{-1} \mathrm{~s}^{-2} \\
f & =1 \mathrm{year}^{-1}=3.170 \times 10^{-8} \mathrm{~Hz} \\
H_{0} & =h \cdot 100 \mathrm{~km} \mathrm{~s}^{-1} \mathrm{Mpc}^{-1} \\
& =h \cdot 3.24078 \times 10^{-18} \mathrm{~Hz},
\end{aligned}
$$

which yields

$$
\begin{aligned}
h_{c, 1}= & \frac{7.37 \times 10^{-17}}{\sqrt{h}}\left\langle\left(\frac{M_{c}}{10^{7} M_{\odot}}\right)^{5 / 3}\right\rangle^{1 / 2} \\
& \times\left(\frac{R_{0}}{10^{-4} \mathrm{Gyr}^{-1} \mathrm{Mpc}^{-3}}\right)^{1 / 2} I^{1 / 2} .
\end{aligned}
$$

Parameterized in this way, the unknowns in the predicted amplitude of the strain spectrum are the Hubble constant, the average chirp mass, the local rate of coalescing binary systems, and the factor $I$ as it is defined in Equation (20). This factor encompasses various redshift-evolving values, and can be broken down into more physical direct parameters, depending on the input parameterization being considered. Note that below, we set $h=1$.

\subsubsection{Implications of our Strain Amplitude Limit}

As implied above, a limit from pulsar timing on $h_{c}(f)$ can bound a number of parameters of astrophysical significance. However, given the sizable unknowns in these parameters, the precise implications of our $A_{1}$ limit will depend on the model being considered. The parameterization of Jaffe \& Backer (2003) provided an estimate of $\left\langle M_{c}^{5 / 3}\right\rangle=\left(2.3 \times 10^{7} M_{\odot}\right)^{5 / 3}$, extrapolating this value from the locally observed relationship between spheroid mass and black hole mass (Merritt \& Ferrarese 2001) and various theoretical predictions for the spheroid mass function. They also employ $R_{0}=9 \times 10^{-5} \mathrm{Gyr}^{-1} \mathrm{Mpc}^{-3}$. We thus place a limit of $I \leqslant 2646$ using these values. Jaffe \& Backer (2003) parameterize the redshift dependence of merger rate evolution as $I \propto R(z) \propto(1+z)^{\gamma}$, where $\gamma$ is expected to range between -0.4 to 2.3 . Using this parameterization and the cosmological parameters noted above, we constrain the redshift dependence of $R(z)$ to $\gamma \lesssim 7$. Thus, we do not yet place physically interesting constraints on this value.
Most recently, Sesana et al. (2008) considered a broad range of probable parameter uncertainty values applied to four leading scenarios for black hole growth in the context of hierarchical merging. Of most importance here, they found that when accounting for uncertainties in all model parameters, the estimated range of predicted $A_{1}$ values produced a relatively narrow predicted amplitude range of $1 \times 10^{-16} \lesssim A_{1} \lesssim$ $3 \times 10^{-15}$. While we are not yet sensitive enough to impact the upper boundary of their predicted range, it is pertinent to note that our current sensitivity is approximately a factor of two away from the expected highest prediction. If adequate GW sensitivity can be achieved, pulsar timing will probe quantities not readily accessible by electromagnetic observations. Following from the above discussion, the largest uncertainties noted by Sesana et al. (2008) were the SMBH mass function (which, if constrained, leads to limits on galactic host/black hole mass relationships, particularly in the most massive regime) and the local merger rate of massive galaxies. They also considered the amount of merger-induced accretion onto black holes and the post-merger black hole inspiral rate, finding these to be likewise poorly constrained.

The other pertinent finding of Sesana et al. (2008) was that with a paucity depending on the input model, GW spectral bins above $\sim 1-5 \times 10^{-8} \mathrm{~Hz}$ were populated by increasingly few black holes-at the highest frequencies, sometimes one or none. A lack of black holes contributing to the high-frequency GW spectrum could affect us in two ways. First, it could change the observed GWB spectral slope; in fact, Sesana et al. used an expanded version of our Equation (1) to include a break in the spectrum. Alternatively, it could induce non-stochastic signals, requiring a directional/point-source analysis approach to detect the emitted signal. Either of these consequences would corrupt the sensitivity and applicability of the analysis performed here, particularly if the analysis were performed on data spans of $<3$ years. All but one of the pulsar data sets presented here exceed this length, thus the influence of this issue is expected to be minimal. As the contributing population is expected to increase at lower frequencies, the impact of this problem will decrease as further data are collected on these pulsars, and as we reach the aforementioned sensitivity improvements that come with increased data span. Nonetheless, further studies must be performed to address the effects of the $\mathrm{N}$-source contribution issue, particularly to understand its effect on the expected Hellings-Downs function.

\subsection{Cosmic Strings}

Cosmic strings or superstrings are linear-dimensional structures proposed to arise due to phase transitions in the early universe. Networks of cosmic strings can generate powerful bursts of GW radiation through the production of cusps on long strings and loops, leading to a stochastic GWB with $\alpha \simeq-7 / 6$ in the nanohertz frequency band (e.g., Damour \& Vilenkin 2005; Siemens et al. 2007). The amount of GW produced depends on physical parameters such as the string loop size scale, the reconnection probability and the string tension. Conversely, measured GW limits constrain the possible values of these parameters. Following the analysis of Siemens et al. (2007), our results for cosmic strings take the form of sections of cosmic string parameter space that are ruled out or allowed by our upper limit on the gravitational wave background.

In the case where cosmic string loop sizes are set by gravitational back reaction ("small loop" case) we explore the threedimensional parameter space of cosmic string dimensionless 


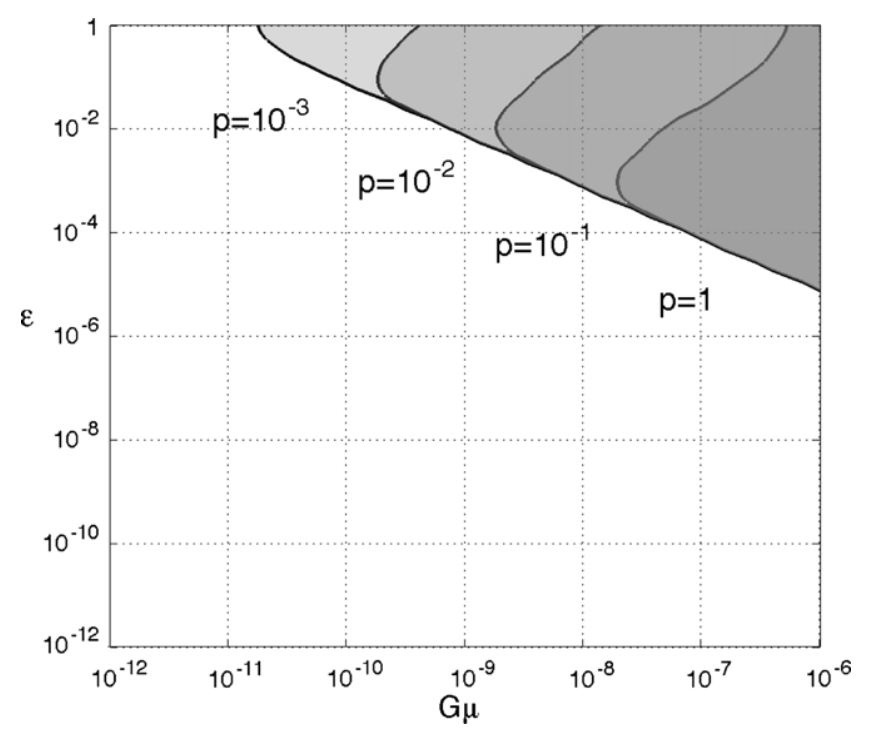

Figure 26. Cosmic string parameter space constraints from our measurement, in the small loop case. The shaded areas shown regions of string tension $(G \mu)$ and loop size $(\varepsilon)$ that are ruled out by our measurement for various values of reconnection probability $(p)$.

tension $G \mu$, reconnection probability $p$, and loop sizes parameterized by $\varepsilon$ (for details, see Siemens et al. 2007). The shaded areas of Figure 26 show the regions of the $\varepsilon-G \mu$ plane ruled out for four values of the reconnection probability $p=1,10^{-1}$, $10^{-2}$, and $10^{-3}$. For example, for $\varepsilon=1$, and $p=10^{-3}$ the string tension $G \mu$ is constrained to be less than about $2 \times 10^{-11}$.

If the size of cosmic string loops is instead given by the large-scale dynamics of the network, as suggested by recent numerical simulations (Blanco-Pillado et al. 2011), we fix the cosmic string loop length and explore the two-dimensional parameter space of reconnection probability and string tension following Siemens et al. (2007). These results are shown in Figure 27. The stochastic background produced in these models is substantially larger and therefore our constraints on parameters are correspondingly tighter. For example for a reconnection probability $p=1, G \mu<10^{-9}$, and for a reconnection probability of $p=10^{-3}$ all cosmic string tensions above $10^{-12}$ are ruled out.

\subsection{Future Performance}

Predicting the future sensitivity of a PTA project to GW depends on a large number of poorly constrained factors. These include the level of pulsar-intrinsic timing noise in the MSP population (Shannon \& Cordes 2010), the statistical characteristics of the pulsar radio emission (Osłowski et al. 2011), and the influence of the interstellar medium on pulsar timing (Coles et al. 2010). A detailed assessment of the effect of various kinds of noise on GW detection sensitivity was done by Cordes \& Shannon (2012). Assuming that all factors besides experiment duration $T$ (e.g., number of pulsars, observing cadence, telescope instrumentation, etc.) remain the same, we can roughly bound our future GW limits by two cases:

1. Most optimistically, the data could remain dominated primarily by "white" radiometer noise. In this case, limits on $A_{1}$ scale in proportion to $T^{\alpha-3 / 2}$. For example, $A_{1} \propto$ $T^{-13 / 6}$ for the SMBH GWB. With an additional five years of data, we will reach $A_{1} \sim 1.6 \times 10^{-15}$ for this spectrum, well into the range of expected $\mathrm{GW}$ amplitudes.

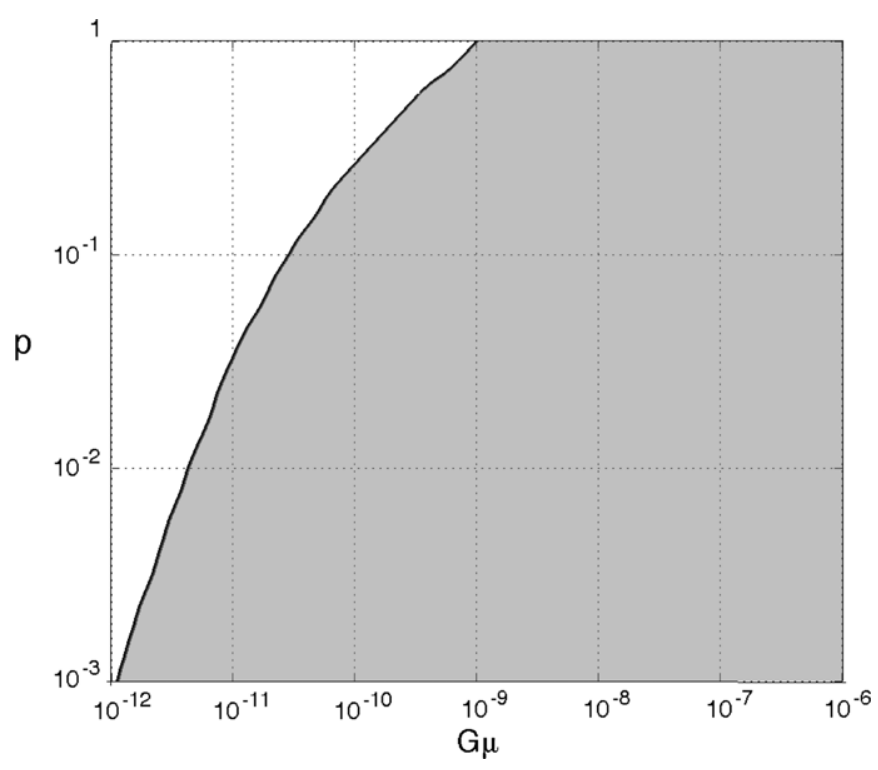

Figure 27. Cosmic string constraints in the large loop case, in terms of string tension $G \mu$ and reconnection probability $p$. The shaded area is ruled out by our GW upper limit.

2. On the other hand, if the data are completely dominated by timing noise with spectral index equal to or steeper than the GW signal, limits improve only as $T^{-1 / 2}$. Doubling the data span would only improve the result to $A_{1} \sim 5 \times 10^{-15}$. Note that this does not appear to be the situation currently for the majority of our pulsars.

Intermediate cases, which are more likely to apply in reality than either of the two extremes above, include those where only some of the pulsars are timing-noise dominated, or have timing noise that is less red than the GW signal. Note that these scalings only apply for GW limits (that is, non-detections) - in the GW signal-dominated regime, and during the transition from limit to detection, different rules apply (see Cordes \& Shannon 2012). These estimates also do not take into account any future improvements in the experiment. In reality both telescopes have recently undergone a major upgrade in digital pulsar instrumentation with the installation of the GUPPI and PUPPI pulsar backends (P. B. Demorest et al. 2012, in preparation). These provide an order of magnitude more radio bandwidth than was used for this analysis. NANOGrav is also currently in the process of expanding the number of pulsars monitored to 230 , as new MSPs are discovered by searches including Fermi gamma-ray source follow-up (e.g., Ransom et al. 2011), the Arecibo PALFA survey (Lazarus et al. 2012), and the Green Bank North Celestial Cap survey (K. Stovall et al. 2012, in preparation). Additional improvements may come from connecting our current data set to historical pulsar timing data stretching back as far as 20 years for some sources.

\section{CONCLUSIONS}

In this paper, we presented and analyzed five years of radio timing data on 17 pulsars taken with the two largest singledish telescopes in the world. Our timing analysis included novel methods for dealing with time-variable dispersion measure and intrinsic profile shape evolution with frequency. We achieved sub-microsecond rms timing results on all but two of our sources, and rms residuals of only $\sim 30-50 \mathrm{~ns}$ in the two best cases. We presented a new time-domain method for detecting and/or limiting timing fluctuations of a known spectral shape 
but unknown amplitude. The key feature of this analysis was proper accounting for the signal power removed by the timing model fit, without requiring dependence on simulation for this step. Applied to our timing of $\mathrm{J} 1713+0747$, these methods set a single-pulsar limit on the stochastic gravitational wave background amplitude of $A_{1}<1.1 \times 10^{-14}(95 \%)$ for the expected SMBH GWB spectrum with spectral index $\alpha=-2 / 3$. We discussed how to measure cross-correlations between the timing of pairs of pulsars while also accounting for both the timing model fit and the presence of correlated (non-white) noise in the data. For $\alpha=-2 / 3$, the measured cross-correlations in our data set constrain $A_{1}^{2}$ to $(-10 \pm 26) \times 10^{-30}$, or alternately a $2 \sigma$ upper limit of $A_{1}<7.2 \times 10^{-15}$. We discussed how our measurement will improve with time, and suggest that prospects are good for obtaining astrophysically constraining GW limits, or possibly even a detection, over the next five years.

The NANOGrav project receives support from the National Science Foundation (NSF) PIRE program award number 0968296. NANOGrav research at UBC is supported by an NSERC Discovery Grant and Discovery Accelerator Supplement. P.B.D. acknowledges support from a Jansky Fellowship of the National Radio Astronomy Observatory during 2007-2010. A.N.L. gratefully acknowledges the support of NSF grant AST CAREER 07-48580. Part of this research was carried out at the Jet Propulsion Laboratory, California Institute of Technology, under a contract with the National Aeronautics and Space Administration. The National Radio Astronomy Observatory is a facility of the NSF operated under cooperative agreement by Associated Universities, Inc. The Arecibo Observatory is operated by SRI International under a cooperative agreement with the NSF (AST-1100968), and in alliance with Ana G. Méndez-Universidad Metropolitana, and the Universities Space Research Association. The authors thank Rutger van Haasteren for helpful discussions about his previous work on this topic.

\section{REFERENCES}

Anholm, M., Ballmer, S., Creighton, J. D. E., Price, L. R., \& Siemens, X. 2009, PhRvD, 79, 084030

Backer, D. C., Kulkarni, S. R., Heiles, C., Davis, M. M., \& Goss, W. M. 1982, Natur, 300, 615

Blanco-Pillado, J. J., Olum, K. D., \& Shlaer, B. 2011, PhRvD, 83, 083514

Blandford, R., Romani, R. W., \& Narayan, R. 1984, JApA, 5, 369

Coifman, R. R., \& Donoho, D. L. 1995, in Wavelets and Statistics, ed. A. Antoniadis \& G. Oppenheim (Lecture Notes in Statistics, Vol. 103; Berlin: Springer), 125

Coles, W., Hobbs, G., Champion, D. J., Manchester, R. N., \& Verbiest, J. P. W. 2011, MNRAS, 418, 561
Coles, W. A., Rickett, B. J., Gao, J. J., Hobbs, G., \& Verbiest, J. P. W. 2010, ApJ, 717, 1206

Cordes, J. M., \& Shannon, R. M. 2012, ApJ, 750, 89

Damour, T., \& Deruelle, N. 1985, AnIHP, 43, 107

Damour, T., \& Vilenkin, A. 2005, PhRvD, 71, 063510

Demorest, P. B. 2007, PhD thesis, Univ. California

Detweiler, S. 1979, ApJ, 234, 1100

Ferdman, R. 2008, PhD thesis, Univ. British Columbia

Foster, R. S., \& Backer, D. C. 1990, ApJ, 361, 300

Gonzalez, M. E., Stairs, I. H., Ferdman, R. D., et al. 2011, ApJ, 743, 102

Grishchuk, L. P. 2005, PhyU, 48, 1235

Guinot, B. 1988, A\&A, 192, 370

Heiles, C., Perillat, P., Nolan, M., et al. 2001, PASP, 113, 1274

Hellings, R. W., \& Downs, G. S. 1983, ApJL, 265, 39

Hobbs, G., Jenet, F., Lee, K. J., et al. 2009, MNRAS, 394, 1945

Hobbs, G. B., Edwards, R. T., \& Manchester, R. N. 2006, MNRAS, 369, 655

Hotan, A. W., Bailes, M., \& Ord, S. M. 2005, MNRAS, 362, 1267

Hotan, A. W., van Straten, W., \& Manchester, R. N. 2004, PASA, 21, 302

Jaffe, A. H., \& Backer, D. C. 2003, ApJ, 583, 616

Jenet, F. A., Hobbs, G. B., Lee, K. J., \& Manchester, R. N. 2005, ApJL, 625, 123

Jenet, F. A., Hobbs, G. B., van Straten, W., et al. 2006, ApJ, 653, 1571

Jenet, F. A., Lommen, A., Larson, S. L., \& Wen, L. 2004, ApJ, 606, 799

Kaspi, V. M., Taylor, J. H., \& Ryba, M. F. 1994, ApJ, 428, 713

Lazarus, P., Allen, B., Bhat, N. D. R., et al. 2012, in Proc. IAUS 291, Neutron Stars and Pulsars: Challenges and Opportunities after 80 Years, ed. J. van Leeuwen (Cambridge University Press), in press (arXiv:1210.8003)

Kramer, M., Lange, C., Lorimer, D. R., et al. 1999, ApJ, 526, 957

Lorimer, D. R., \& Kramer, M. 2004, Handbook of Pulsar Astronomy (Cambridge Observing Handbooks for Research Astronomers, Vol. 4; Cambridge: Cambridge Univ. Press)

Merritt, D., \& Ferrarese, L. 2001, ApJ, 547, 140

Osłowski, S., van Straten, W., Hobbs, G. B., Bailes, M., \& Demorest, P. 2011, MNRAS, 418, 1258

Papoulis, A., \& Pillai, S. U. (ed.) 2002, Probability, Random Variables, and Stochastic Processes (4th ed.; New York: McGraw-Hill)

Peters, P. C., \& Mathews, J. 1963, PhRv, 131, 435

Press, W. H., Teukolsky, S. A., Vetterling, W. T., \& Flannery, B. P. 1992, Numerical Recipes in C. The Art of Scientific Computing (2nd ed.; Cambridge: Cambridge Univ. Press)

Ramchandran, R., Demorest, P., Backer, D. C., Cognard, I., \& Lommen, A. 2006, ApJ, 645, 303

Ransom, S. M., Ray, P. S., Camilo, F., et al. 2011, ApJL, 727, 16

Rickett, B. J. 1990, ARA\&A, 28, 561

Sazhin, M. V. 1978, SvA, 22, 36

Sesana, A., Vecchio, A., \& Colacino, C. N. 2008, MNRAS, 390, 192

Sesana, A., Vecchio, A., \& Volonteri, M. 2009, MNRAS, 394, 2255

Shannon, R. M., \& Cordes, J. M. 2010, ApJ, 725, 1607

Siemens, X., Mandic, V., \& Creighton, J. 2007, PhRvL, 98, 111101

Taylor, J. H. 1992, RSPTA, 341, 117

Taylor, J. H., \& Weisberg, J. M. 1989, ApJ, 345, 434

van Haasteren, R., Levin, Y., Janssen, G. H., et al. 2011, MNRAS, 414, 3117

van Haasteren, R., Levin, Y., McDonald, P., \& Lu, T. 2009, MNRAS, 395, 1005

van Straten, W. 2004, ApJS, 152, 129

van Straten, W. 2006, ApJ, 642, 1004

Weisberg, J. M., Nice, D. J., \& Taylor, J. H. 2010, ApJ, 722, 1030

Yardley, D. R. B., Coles, W. A., Hobbs, G. B., et al. 2011, MNRAS, 414,1777 